\title{
In situ formed reactive oxygen species-responsive scaffold with gemcitabine and checkpoint inhibitor for combination therapy
}

\author{
Chao Wang, ${ }^{1,2 *}$ Jinqiang Wang, ${ }^{1,2 *}$ Xudong Zhang, ${ }^{1,2}$ Shuangjiang $\mathrm{Yu}^{1,2}{ }^{1,2}$ Wen ${ }^{1,2}$ Quanyin $\mathrm{Hu}^{1,2}$ \\ Yanqi Ye, ${ }^{1,2}$ Hunter Bomba, ${ }^{1,2}$ Xiuli Hu, ${ }^{1}$ Zhuang Liu, ${ }^{3}$ Gianpietro Dotti, ${ }^{4}$ Zhen Gu $^{1,2,5 t}$ \\ Patients with low-immunogenic tumors respond poorly to immune checkpoint blockade (ICB) targeting the pro- \\ grammed death-1 (PD-1)/programmed death-ligand 1 (PD-L1) pathway. Conversely, patients responding to ICB \\ can experience various side effects. We have thus engineered a therapeutic scaffold that, when formed in situ, \\ allows the local release of gemcitabine (GEM) and an anti-PD-L1 blocking antibody (aPDL1) with distinct release \\ kinetics. The scaffold consists of reactive oxygen species (ROS)-degradable hydrogel that releases therapeutics in \\ a programmed manner within the tumor microenvironment (TME), which contains abundant ROS. We found that \\ the aPDL1-GEM scaffold elicits an immunogenic tumor phenotype and promotes an immune-mediated tumor \\ regression in the tumor-bearing mice, with prevention of tumor recurrence after primary resection.
}

\section{INTRODUCTION}

Immune checkpoint blockade (ICB) targeting the programmed death-1 (PD-1)/programmed death-ligand 1 (PD-L1) pathway $(1,2)$ induces remarkable clinical responses in various malignancies $(3,4)$, including melanoma, non-small cell lung, kidney, head and neck, and bladder cancers (5-7). However, only patients with immunogenic tumors characterized by high neoantigen burden (8), preinfiltration of effector T cells (9), and expression of PD-L1 $(10,11)$ seem to achieve durable clinical responses after the administration of ICB $(12,13)$. Moreover, clinical application of ICB has also been associated with various side effects in normal organs (14-16). On the basis of these studies, strategies aimed at promoting an immunogenic tumor phenotype, increasing ICB response, and avoiding severe side effects remain a central theme in the field of cancer immunotherapy (17).

Previous reports showed that prior chemotherapy enhanced the therapeutic outcome of immunotherapy (18-22), which also reversed chemoresistance after prolonged chemotherapy $(20,23)$. Although some chemotherapeutic drugs have modest activity when used as single treatments, their combination with immunotherapy may result in enhanced anticancer effects and can be used to promote an immunogenic tumor phenotype (24-26). In addition, engineered delivery vehicles and scaffolds are increasingly considered as promising tools for transporting immunotherapeutics (27-33), with decreased systemic toxicities. In addition, the regulated release of payloads and the kinetics of the degradation of the supporting matrix upon in vivo administration are aspects particularly relevant for the treatment efficacy $(27,33)$.

\footnotetext{
${ }^{1}$ Joint Department of Biomedical Engineering, University of North Carolina at Chapel Hill and North Carolina State University, Raleigh, NC 27695, USA. 'Division of Pharmacoengineering and Molecular Pharmaceutics and Center for Nanotechnology in Drug Delivery, Eshelman School of Pharmacy, University of North Carolina at Chapel Hill, Chapel Hill, NC 27599, USA. Institute of Functional Nano and Soft Materials, Jiangsu Key Laboratory for Carbon-Based Functional Materials and Devices, Soochow University, Suzhou 215123, China. ${ }^{4}$ Department of Microbiology and Immunology, University of North Carolina at Chapel Hill, Chapel Hill, NC 27599, USA. ${ }^{5}$ Department of Medicine, University of North Carolina at Chapel Hill, Chapel Hill, NC 27599, USA.

${ }^{*}$ These authors contributed equally to this work.

tCorresponding author. Email: zgu@email.unc.edu
}

Here, we have generated an in situ formed bioresponsive scaffold suitable for localized chemoimmunotherapy, in which gemcitabine (GEM) and scaffold enhance an immunogenic tumor phenotype and ICB promotes subsequent therapeutic immune response (Fig. 1A). We hypothesized that injectable reactive oxygen species (ROS)responsive hydrogel could be used to load and release therapeutics upon implantation into the tumor site because of highly abundant ROS, which are expressed within the tumor microenvironment (TME) and promote cancer development as well as progression (34-37). Here, we show that a clinically relevant prototype of ROS-degradable hydrogel scaffold promotes an immunogenic tumor phenotype via local GEM delivery and enhances antitumor responses through local release of anti-PD-L1 blocking antibody (aPDL1) in the B16F10 melanoma and 4T1 breast tumor [relatively low-immunogenic (38)] mouse models. Therapeutic advantage of this chemoimmunotherapy is also demonstrated by the prevention of tumor recurrence after primary resection.

\section{RESULTS}

\section{Synthesis and characterization of in situ formed bioresponsive scaffold}

ROS-responsive hydrogel was obtained by crosslinking poly(vinyl alcohol) (PVA) with a ROS-labile linker: $N^{1}$-(4-boronobenzyl)- $N^{3}-(4$ boronophenyl)- $N^{1}, N^{1}, N^{3}, N^{3}$-tetramethylpropane-1,3-diaminium (TSPBA) (fig. S1), which was synthesized via quaternization reaction of $N^{1}, N^{1}, N^{3}, N^{3}$-tetramethylpropane-1,3-diamine with an excess of 4-(bromomethyl)phenylboronic acid (fig. S2). TSPBA contains two phenylboronic acids that complex with diols on PVA $(39,40)$. Formation of PVA-TSPBA hydrogel was further confirmed by a rheology test (fig. S3). Addition of TSPBA to PVA solution rapidly increased the elastic modulus $\left(\mathrm{G}^{\prime}\right)$, demonstrating the formation of a network between the PVA chains (fig. S3, A to D). The hydrogel can be quickly formed after mixing the PVA and linkers (Fig. 1A and fig. S3, E and F). In vivo gel formation and degradation were then examined in healthy mice. The integrity of the gels remained up to 7 days, whereas the overall size of gels decreased gradually. At week 3 after injection, most gels were degraded at the injection sites (fig. S4), indicating their biodegradability. 
Fig. 1. Schematic and characterization of in situ formed ROS-responsive gel scaffold. (A) Schematic of combination chemoimmunotherapy using a ROS-degradable hydrogel scaffold to deliver GEM and aPDL1 into the TME. (B) Representative Cryo-SEM image of gel scaffold loaded with GEM and aPDL1. Scale bar, $0.5 \mu \mathrm{m}$. Inset: Zoomedin image of the scaffold. Scale bar, $0.1 \mu \mathrm{m}$. (C) Representative fluorescent images of a cryosection of hydrogel in which fluorescein isothiocyanate (FITC) (green) was used as a fluorescent surrogate for GEM and aPDL1 was labeled with Cy5.5 (red). Scale bar, $25 \mu \mathrm{m}$. (D) Morphology changes of hydrogels in $1 \times$ PBS with and without $\mathrm{H}_{2} \mathrm{O}_{2}(1 \mathrm{mM})$ over 7 days. (E and F) Cumulative release profiles of GEM (E) and aPDL1 (F) from hydrogels incubated with PBS with or without $\mathrm{H}_{2} \mathrm{O}_{2}(1 \mathrm{mM})$. Data are means \pm SEM. lgG, immunoglobulin $\mathrm{G}$.

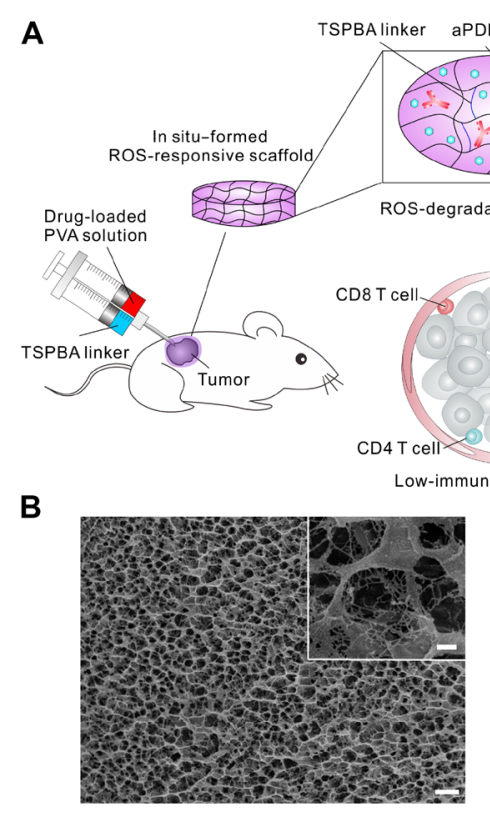

D

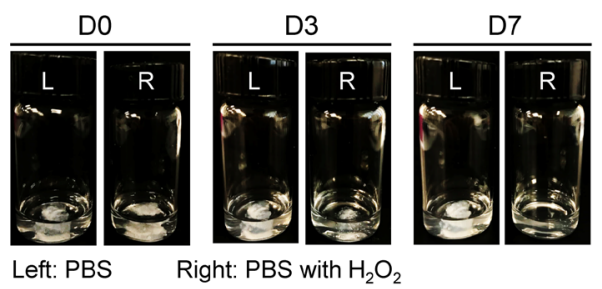

C
E

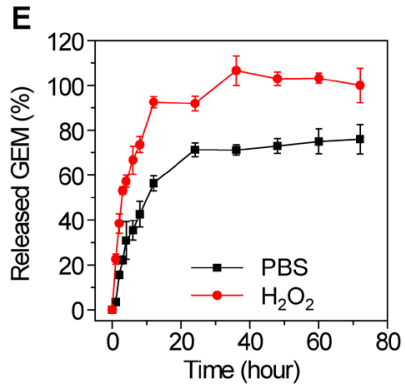

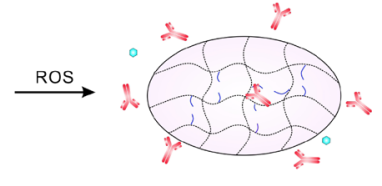

Released aPDL1 Released GEM
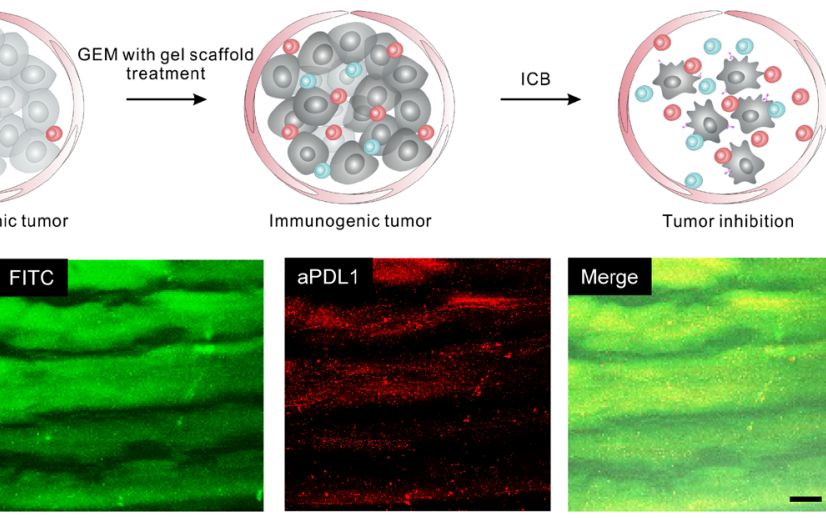

Tumor inhibition

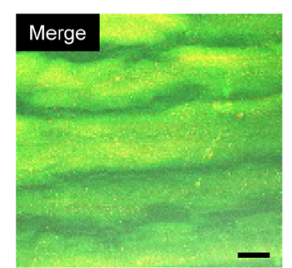

$\mathbf{F}$

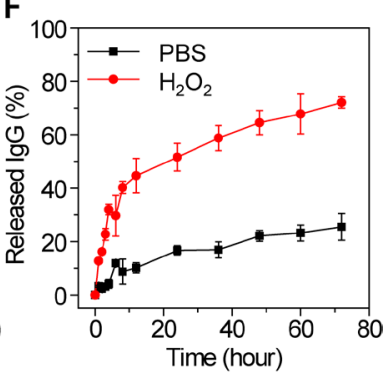

GEM and aPDL1 at therapeutically relevant doses (GEM, $5 \mathrm{mg} / \mathrm{kg}$; aPDL1, $50 \mu \mathrm{g}$ ) were encapsulated into the PVA-TSPBA hydrogel $(200 \mu \mathrm{l} ; 10 \%, \mathrm{w} / \mathrm{w})$. The drug-loaded hydrogels showed similar rheology properties compared with the empty ones (fig. S3, D and F), indicating that the formation of the hydrogel was not significantly affected by the drug encapsulation. The morphology of the dried scaffold network was observed by cryo-scanning electron microscopy (Cryo-SEM), with a porous structure (Fig. 1B). To visualize the distribution of therapeutics in the hydrogels, fluorescein as a fluorescent surrogate for GEM (41) and Cy5.5-labeled aPDL1 were loaded into the hydrogel. In a confocal image of a frozen section of hydrogel, fluorescein displayed a uniform distribution, whereas the dotted signals associated with aPDL1 were detected inside the hydrogel (Fig. 1C).

The TSPBA can be oxidized and hydrolyzed when exposed to $\mathrm{H}_{2} \mathrm{O}_{2}$ in the TME, leading to the dissociation of the polymeric scaffold (fig. S5) and the release of payloads. To verify the ROS-sensitive degradability of the hydrogels, samples were immersed in phosphatebuffered saline (PBS) containing $1.0 \mathrm{mM} \mathrm{H}_{2} \mathrm{O}_{2}$ at $37^{\circ} \mathrm{C}$. Changes in the morphology of the scaffolds were observed over time (Fig. 1D). The release profiles of GEM and aPDL1 were quantified using highperformance liquid chromatography (HPLC) and enzyme-linked immunosorbent assay (ELISA), respectively. As expected, GEM and aPDL1 were released from the hydrogel in $\mathrm{H}_{2} \mathrm{O}_{2}$ solution with an increased rate compared to that in PBS only. Most of the GEM was released within 1 day, whereas aPDL1 showed a more sustained re- lease profile, with $80 \%$ released within 3 days (Fig. 1, E and F). The release pattern was further examined in vivo after the peritumoral injection of the scaffold with payloads in the B16F10 mouse model. Confocal imaging of tumor sections (fig. S6) showed that the fluorescein signal (fluorescent surrogate for GEM) was rapidly evident (within day 1), whereas the signal corresponding to aPDL1 increased gradually within 3 days. These distinct release dynamics facilitated the delivery of GEM and aPDL1 into the TME for the intended sequential effects.

\section{Therapeutic scaffold for eliciting immunogenic tumor phenotype}

Next, we measured the response of immune cells and tumor cells after treatment of scaffold loaded with GEM (GEM@Gel) in vivo. GEM was loaded into the hydrogel and injected peritumorally. We found that a high dose of GEM $(25 \mathrm{mg} / \mathrm{kg})$ within the GEM@Gel significantly $(P=0.0151)$ depleted the tumor-infiltrating lymphocytes (TILs) (fig. S7A) and had no significant effects on the survival (fig. S7, B and C). In contrast, empty hydrogel or a low dose of GEM $(5 \mathrm{mg} / \mathrm{kg}$ ) within the GEM@Gel increased the frequency of TILs at the tumor site (Fig. 2, A and B). To further assess the overall immune effects of the GEM@Gel within the TME, we examined the intratumoral presence of ROS, regulatory $\mathrm{T}$ cells $\left(\mathrm{T}_{\text {regs }}\right)$, myeloid-derived suppressor cells (MDSCs), and tumor-associated macrophages (TAMs). Intratumoral ROS were significantly $(P<0.001)$ reduced after the empty hydrogel or GEM@Gel implantation (fig. S8). Furthermore, 
A
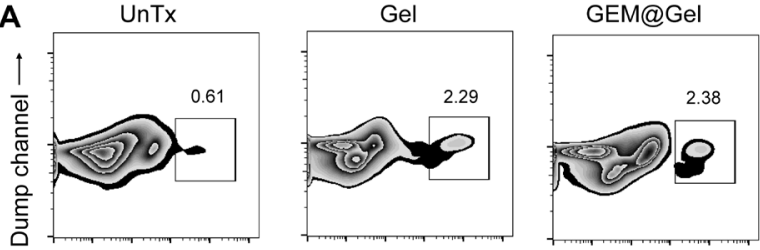

\section{CD45}
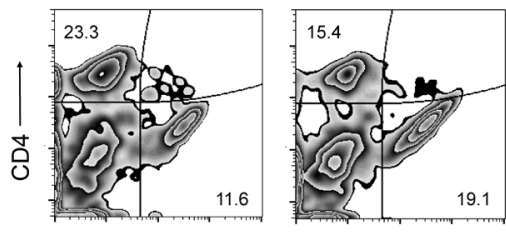

$\mathrm{CD} 8 \longrightarrow$

B
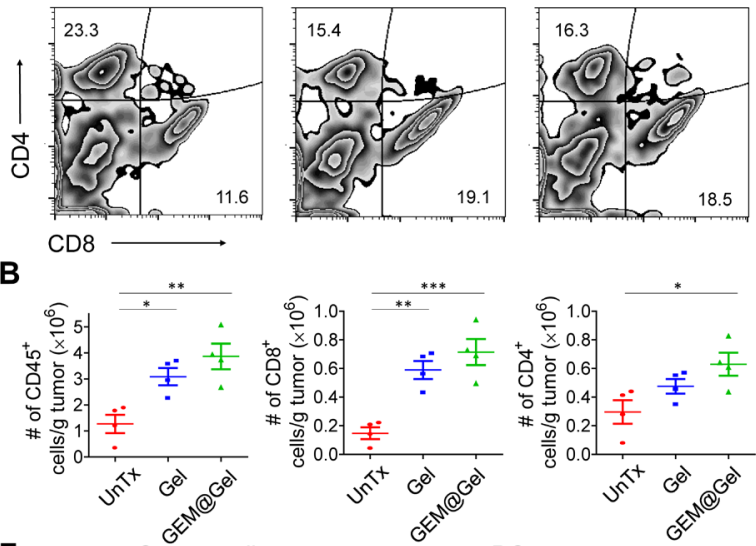

18.5

F
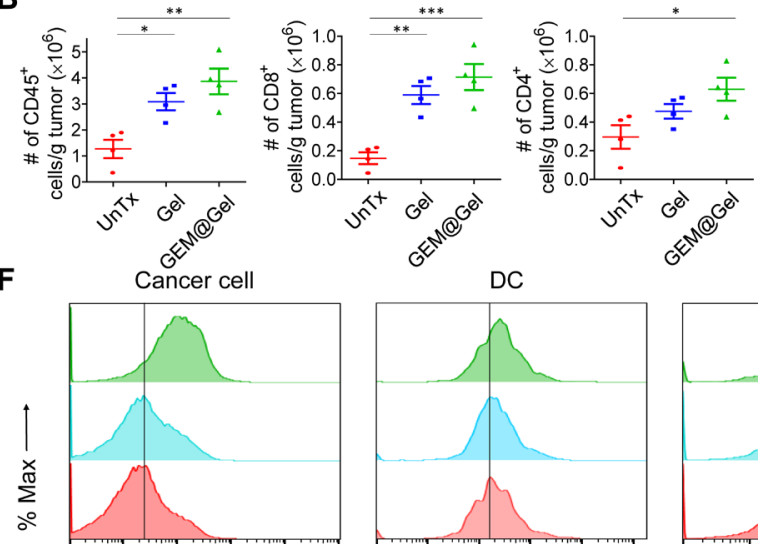
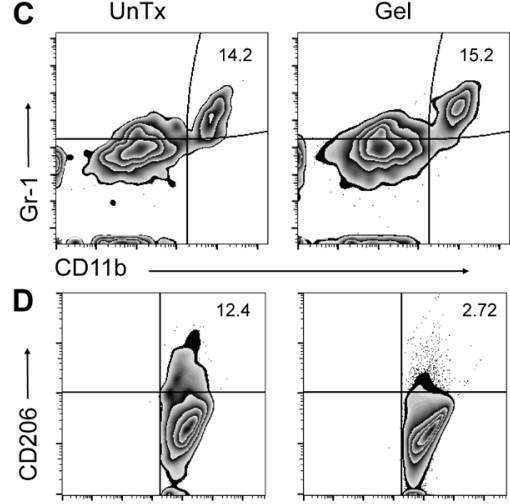

F4/80
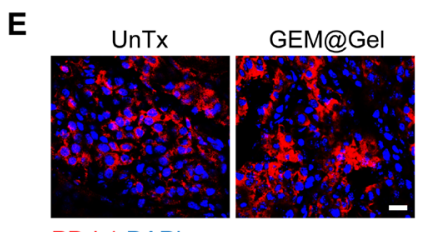

PD-L1 DAPI
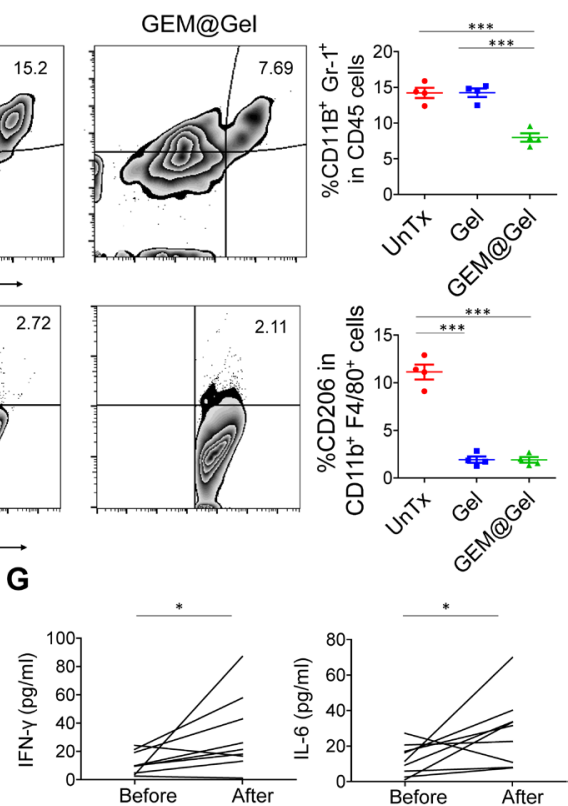

TAM CD4 T cell
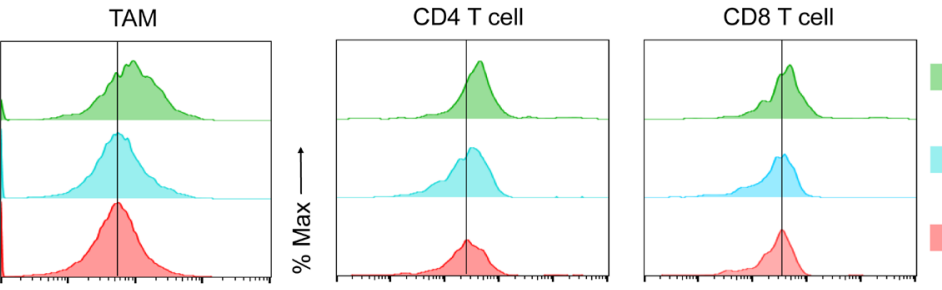

GEM@Gel

Gel

UnTx
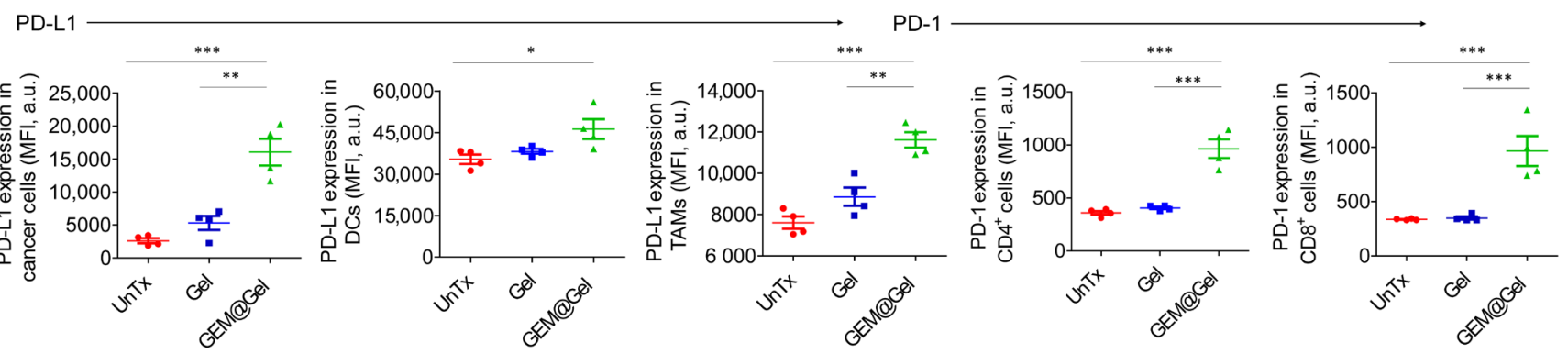

Fig. 2.GEM@Gel implantation for eliciting immunogenic tumor phenotypes. B16F10 tumors harvested from mice implanted with hydrogels or GEM@Gel were analyzed by flow cytometry 2 days after treatment. (A) Representative flow cytometric analysis of T cell infiltration within the tumor and (B) corresponding quantification results. UnTx, untreated. (C) Representative flow cytometric analysis images (left) and the corresponding quantification (right) of MDSCs (CD11 b $\left.\mathrm{b}^{+} \mathrm{Gr}-1^{+}\right)$, gating on $\mathrm{CD} 45^{+}$cells. (D) Representative flow cytometric analysis images (left) and the corresponding quantification (right) of $\mathrm{M} 2$ macrophages $\left(\mathrm{CD} 206^{+}\right)$in $\mathrm{F} 4 / 80^{+} \mathrm{CD} 11 \mathrm{~b}^{+} \mathrm{CD} 45^{+}$cells. (E) Confocal immunofluorescence images of B16F10 tumor with (right) or without (left) GEM@Gel treatment. Red and blue colors represent aPDL1 signals from Cy3conjugated aPDL1 and 4',6-diamidino-2-phenylindole (DAPI) staining of nuclei, respectively. Scale bar, $20 \mu \mathrm{m}$. (F) PD-L1 expression of tumor cells and PD-1 expression of TILs after empty hydrogel or GEM@Gel treatment and the corresponding quantification of PD-L1 and PD-1 mean fluorescence intensity (MFI). a.u., arbitrary units; DC, dendritic cell. (G) Systemic IL-6 and IFN- $\gamma$ concentrations before and after GEM@Gel treatment. Data are means \pm SEM. Statistical significance was calculated by one-way analysis of variance (ANOVA) with Tukey's post hoc test. ${ }^{*} P<0.05 ;{ }^{* *} P<0.01 ;{ }^{* * *} P<0.005$.

although $\mathrm{T}_{\text {regs }}$ were not significantly affected (fig. S9), we observed a significant $(P<0.001)$ reduction of MDSCs $\left(\mathrm{CD} 45^{+} \mathrm{CD} 11 \mathrm{~b}^{+} \mathrm{Gr}-1^{+}\right)$ and M2-polarized TAMs $\left(\mathrm{CD} 20{ }^{\mathrm{hi}} \mathrm{CD} 1 \mathrm{~b}^{+} \mathrm{F} 4 / 80^{+}\right)$(Fig. 2, C and D) (42). A significant $(P<0.001)$ reduction of TAMs was also observed in mice treated with empty hydrogel (Fig. 2D). Together, these data indicate that GEM@Gel enhances the frequency of TILs and reduces immunosuppressive cellular components.

Treatment of B16F10 cancer cells in vitro with GEM resulted in cell death and increased expression of PD-L1 in surviving cells. PD-L1 expression on B16F10 cancer cells was found to be GEM dose- and time-dependent as assessed by flow cytometry, immunofluorescence, and Western blot (fig. S10, A and B). In vivo, GEM@Gel also induced PD-L1 expression on cancer cells, dendritic cells, and macrophages compared to the untreated tumors and tumors treated with empty hydrogels, as well as PD-1 expression in both $\mathrm{CD}^{+}$and $\mathrm{CD} 8^{+}$TILs (Fig. 2, E and F). PD-L1 expression in the tumor cells was increased in a time-dependent manner (fig. S11). Circulating type $1 \mathrm{~T}$ helper $\left(\mathrm{T}_{\mathrm{H}} 1\right)$ cytokines were also measured before and after GEM@Gel 
implantation (Fig. $2 \mathrm{G}$ and fig. S12). Among them, interleukin-6 (IL-6) and interferon- $\gamma($ IFN- $\gamma)$ were significantly $(P<0.05)$ upregulated after GEM@Gel implantation (Fig. 2G), and IFN- $\gamma$ is known to induce PD-L1 expression in tumor cells (43-45). These results substantiate that GEM@Gel can elicit an inflamed and immunogenic TME.

\section{Scaffold-mediated combination therapy}

To validate whether the proposed combination chemoimmunotherapy strategy can promote antitumor effects, we used the B16F10 mouse melanoma tumor model. Tumor-bearing mice were implanted peritumorally with GEM@Gel (200 $\mu \mathrm{l} ; 10 \%$, w/w) (GEM, $5 \mathrm{mg} / \mathrm{kg})$, aPDL1@Gel (aPDL1, $50 \mu \mathrm{g}$ per mouse), or aPDL1-GEM@Gel (aPDL1, $50 \mu \mathrm{g}$ per mouse; GEM, $5 \mathrm{mg} / \mathrm{kg}$ ). Tumor growth was monitored by the bioluminescence signals of B16F10 cells (Fig. 3A). The empty hydrogel and GEM@Gel showed similar effects and were not superior to untreated control. aPDL1@Gel-treated mice showed a delay of tumor growth. In contrast, mice receiving aPDL1-GEM@Gel showed noticeable tumor inhibition effects (Fig. 3, B and C). The tumor sizes in mice correlated with their survival (Fig. 3D). Fifty percent of mice survived at least 60 days after treatment with aPDL1-GEM@Gel. In contrast, none of the mice survived in any of the control groups after 2 months (Fig. 3D). We compared aPDL1-GEM@Gel with nonencapsulated GEM and aPDL1. aPDL1-GEM@Gel treatment was superior in inhibiting tumor growth compared to nonencapsulated drugs delivered locally or systemically (fig. S13).

Furthermore, tumors were harvested and analyzed by immunofluorescence and flow cytometry on day 10 after treatments. aPDL1GEM@Gel-treated mice showed marked infiltration with CD8 ${ }^{+}$ and $\mathrm{CD} 4^{+} \mathrm{T}$ cells compared to the untreated mice (Fig. 3E). Tumor weights were also significantly $(P<0.001)$ lower in the aPDL1GEM@Gel-treated mice on day 10 (fig. S14), which paralleled an increase in absolute numbers of TILs (fig. S14). More strikingly, the absolute number of $\mathrm{CD}^{+} \mathrm{T}$ cells per gram of tumor increased by more than 20-fold in the aPDL1-GEM@Gel-treated mice compared with the untreated control and 2.5-fold over that of the
A

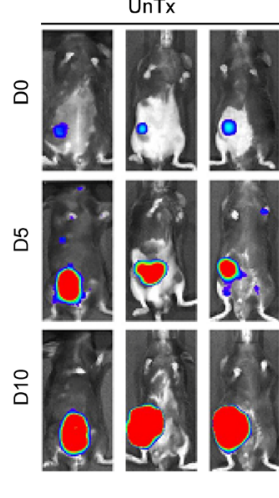

Gel

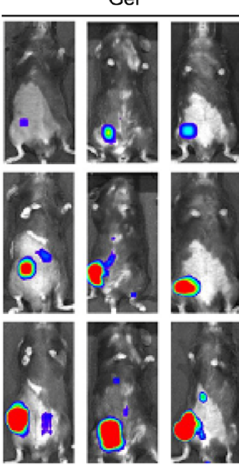

GEM@Gel

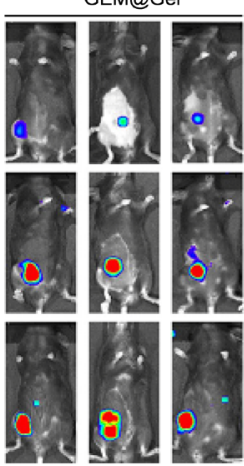

aPDL1@Gel

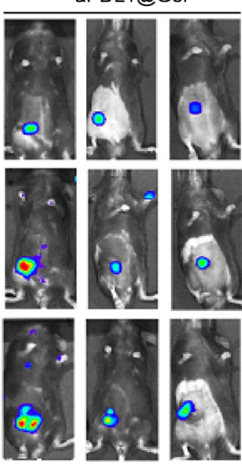

aPDL1-GEM@Gel

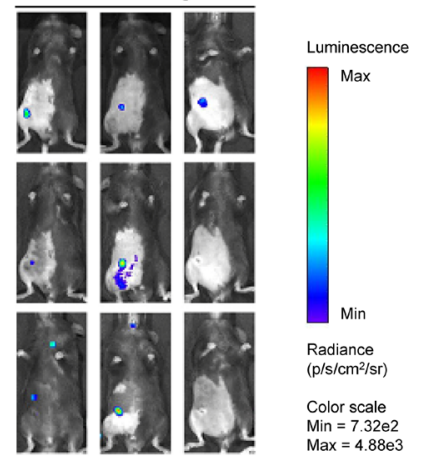

B

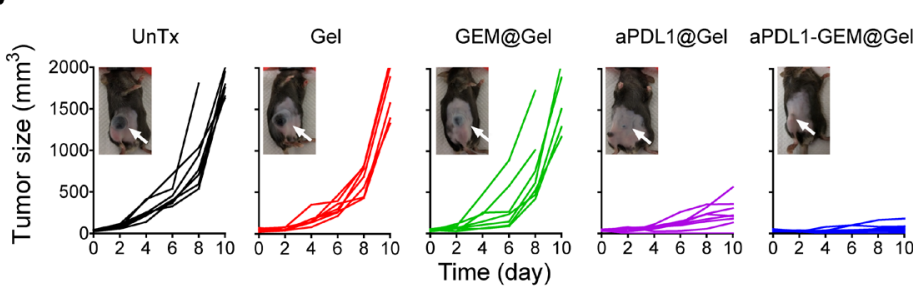

E

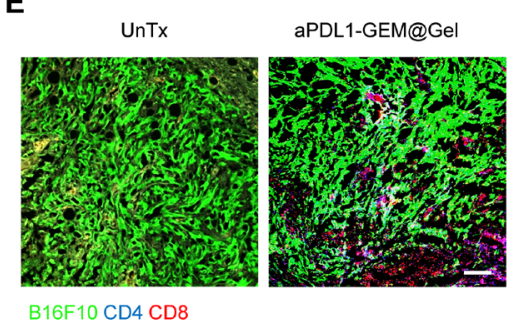

$\mathbf{F}$

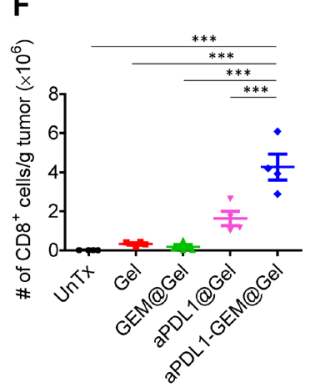

G

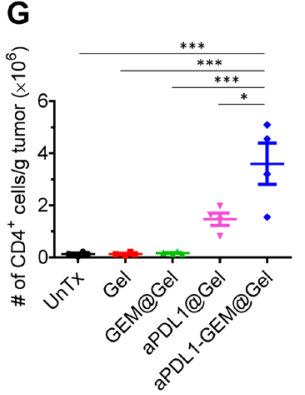

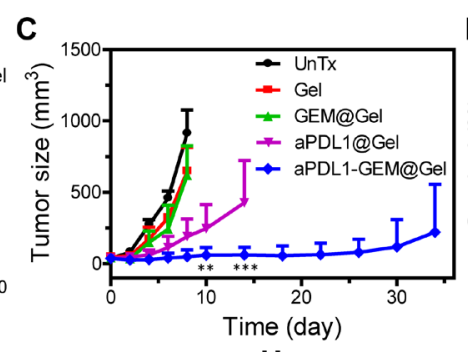

$\mathbf{H}$

D

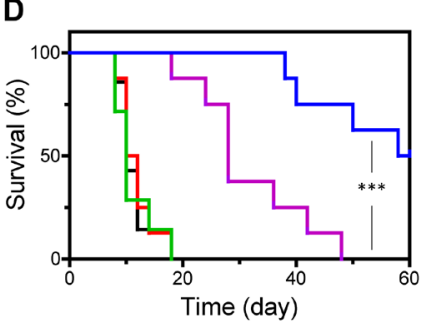

I
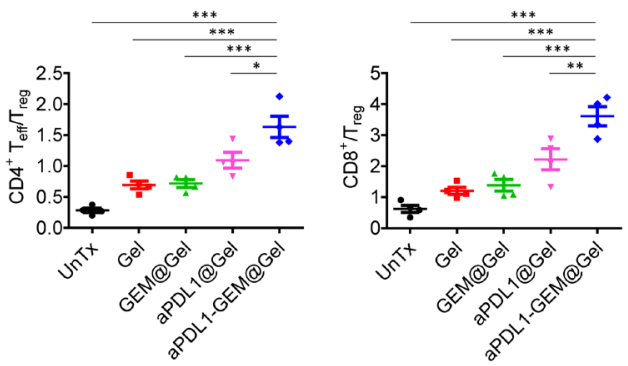

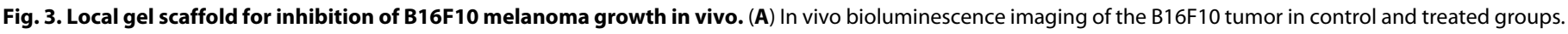

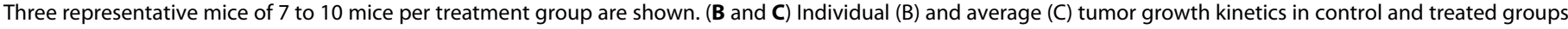

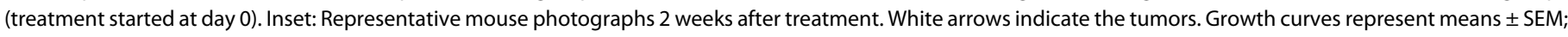

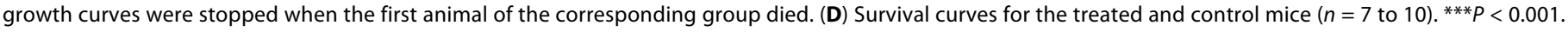

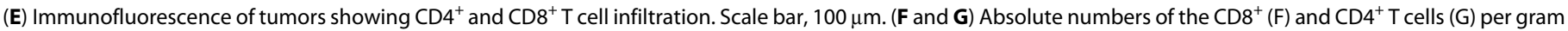

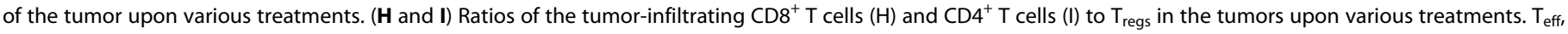
effector T cell. Data are means \pm SEM. Statistical significance was calculated by one-way ANOVA with Tukey's post hoc test. ${ }^{*} P<0.05$; ${ }^{* *} P<0.01 ;{ }^{* * *} P<0.005$. 
aPDL1@Gel-treated mice (Fig. 3F and fig. S14). In addition, the intratumoral ratios of effector $\mathrm{T}$ cells to $\mathrm{T}_{\text {regs }}$ were increased in mice after aPDL1-GEM@Gel therapy (Fig. 3, G to I, and fig. S14). Together, these observations suggest that aPDL1-GEM@Gel implantation triggers a robust $\mathrm{T}$ cell-mediated antitumor immune response.

To assess whether the local delivery of aPDL1-GEM@Gel also induces systemic immune responses, tumor cells were inoculated on the opposite site of the primary tumor in which the hydrogel was implanted (Fig. 4A). We observed that PD-L1 was up-regulated by tumor cells at both tumor sites compared to tumors in the untreated mice (Fig. 4, B and C). This could be explained by the systemic dis- tribution of cytokines locally induced by aPDL1-GEM@Gel implant (Fig. 2G). For example, blocking systemic IFN- $\gamma$ by a neutralizing antibody decreased the PD-L1 up-regulation in the distant tumor (fig. S15). Furthermore, tumor bioluminescence (Fig. 4, $\mathrm{D}$ and $\mathrm{E})$ and tumor weight (Fig. $4 \mathrm{~F})$ significantly $(P=0.0029)$ decreased in both hydrogel-implanted and untreated tumor sites (Fig. 4G), with corresponding increased infiltration of $\mathrm{CD}^{+} \mathrm{CD}^{+}$ $\mathrm{T}$ cells compared to the untreated control mice (Fig. 4, $\mathrm{H}$ and I). $\mathrm{T}$ cell memory response was also generated in mice treated with aPDL1-GEM@Gel, as they showed increased frequency of endogenous $\mathrm{CD} 44^{+} \mathrm{CD} 122^{\text {hi }}$ central memory $\mathrm{T}$ cells $(46-50)$ in the spleen
A

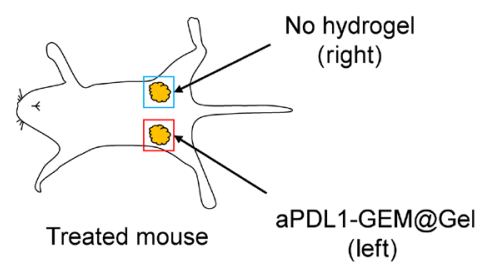

(left)
B

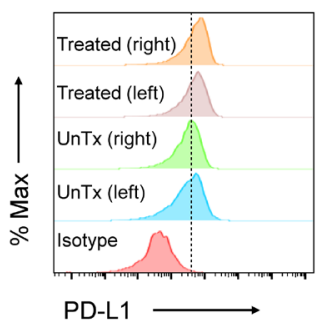

C

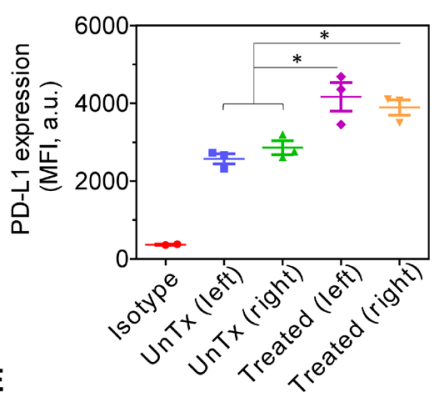

D

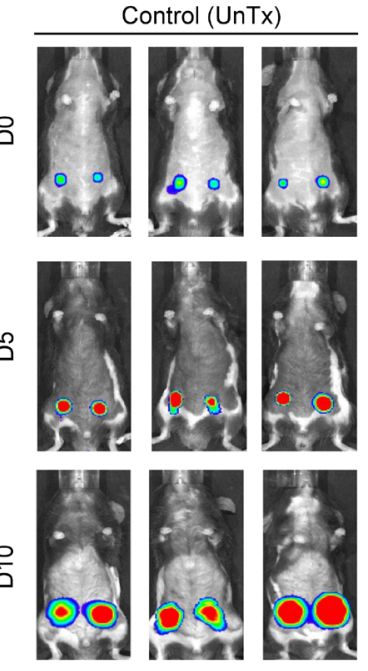

aPDL1-GEM@Gel (treated)
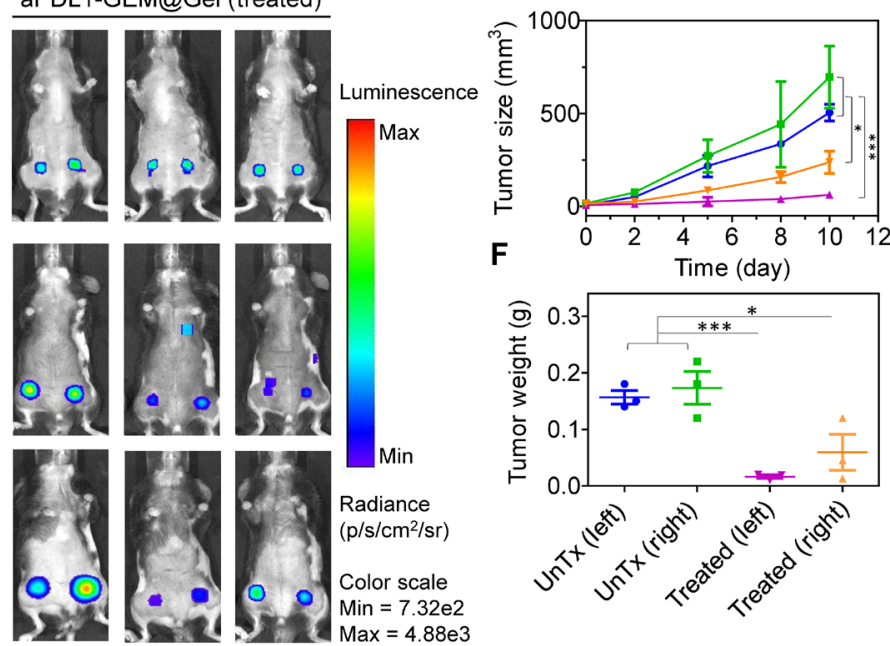

$\rightarrow$ UnTx (left)

- UnTx (right)

* Treated (left)

* Treated (right)
H

UnTx (right)

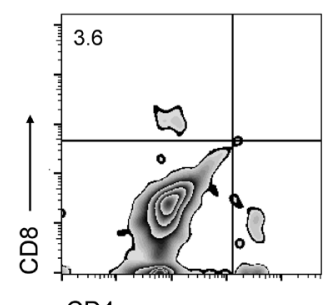

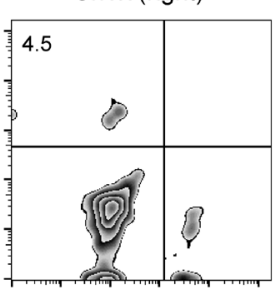

Treated (left)

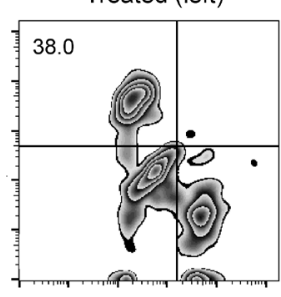

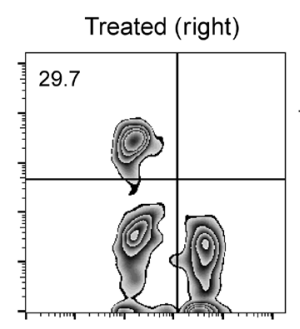

G

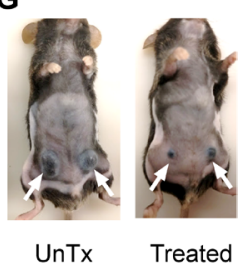

CD4

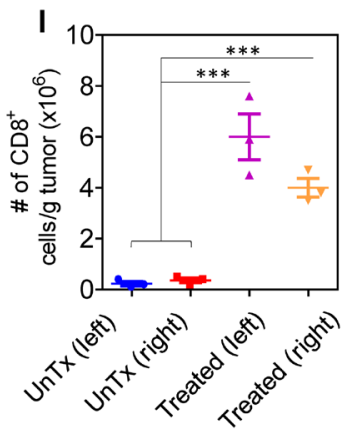

Fig. 4. Local gel scaffold for systemic anticancer immune response. (A) Mice were inoculated with tumor cells in the right and left flanks. Control mice were untreated, whereas treated mice were implanted with hydrogels only on the left flank. (B) PD-L1 expression of cancer cells collected from the tumor sites of the control and treated mice and (C) corresponding quantification of PD-L1 MFI $(n=3)$. (D) In vivo bioluminescence imaging of B16F10 tumors in response to local aPDL1-GEM@Gel treatment. (E) Left and right tumor growth curves and (F) tumor weight on day 10 in untreated and treated mice. (G) Representative photographs of mice on day 10 after treatment. White arrows indicate the tumors. (H) Percentages and representative dot plots of $\mathrm{CD} 4^{+}$and $\mathrm{CD} 8^{+} \mathrm{T}$ cells in tumors of control and treated mice and (I) absolute numbers of $\mathrm{CD} 8{ }^{+}$cells per gram of tumor. Data are means \pm SEM. Statistical significance was calculated by one-way ANOVA with Tukey's post hoc test. ${ }^{*} P<0.05 ;{ }^{* * *} P<0.005$. 
as compared to the control groups (Fig. 5, A to C). When rechallenged with tumor cells, mice that had no detectable tumors after treatment with aPDL1-GEM@Gel did not show significant regrowth of tumor, as indicated by bioluminescence imaging of the lungs (Fig. 5D), photographs of the lungs (Fig. 5E), and hematoxylin and eosin (H\&E) staining (Fig. 5F). Overall survival was also significantly $(P=0.0039)$ better compared to the control group (Fig. 5G). These results indicate that systemic antitumor effects and memory $\mathrm{T}$ cell formation can be achieved by local treatment with aPDL1GEM@Gel.

To further demonstrate that our proposed chemoimmunotherapy could be broadly applicable, we implemented the 4T1 tumor model of triple-negative breast cancer. 4T1 cells were reported to express low amounts of PD-L1 (38). In vitro experiments showed that GEM induced PD-L1 expression in 4T1 cancer cells (fig. S16). For in vivo study, 4T1 cells were inoculated subcutaneously into the right flank of female BALB/c mice. After 14 days, the mice bearing $4 \mathrm{~T} 1$ tumors were treated with GEM@Gel $(200 \mu \mathrm{l} ; 10 \%$, w/w) (GEM, $5 \mathrm{mg} / \mathrm{kg})$, aPDL1@Gel (aPDL1, $50 \mu \mathrm{g}$ per mouse), or aPDL1-GEM@Gel (aPDL1, $50 \mu \mathrm{g}$ per mouse; GEM, $5 \mathrm{mg} / \mathrm{kg}$ ). Encouragingly, we found that aPDL1-GEM@Gel promoted anticancer effects in 4T1-bearing mice as indicated by the bioluminescence imaging (Fig. 6A). Tumor growth was suppressed in mice treated with aPDL1-GEM@Gel (Fig. 6B), which also prolonged survival (Fig. 6C).

Toxic effects always need serious concern when studying combination therapies. PVA with high molecular weight is considered to be highly biologically compatible and eliminated from the body via biliary excretion $(51,52)$. Body weights of mice were not significantly affected after receiving hydrogels loaded with GEM and aPDL1 (fig. S17A). In addition, histology analysis of organs obtained from mice 40 days after treatment indicated no appreciable abnormality or noticeable organ damage (fig. S17B).

\section{Therapeutic scaffold for prevention of postsurgical tumor recurrence}

To further investigate the potency of the in situ formed ROS-responsive scaffold, we performed experiments in a B16F10 incomplete tumor resection model $(53,54)$. ROS-responsive scaffolds containing GEM, aPDL1, or both were directly injected into the resection cavity. Systemically or locally administered nonencapsulated drugs were also included as control groups. Mice receiving aPDL1-GEM@Gel were more protected from local tumor recurrence (with 30\% tumor recurrence rate) (Fig. 7, A to D), with significantly $(P<0.001)$ higher survival rate compared to other groups (Fig. 7E). The body weights
Fig. 5. Local gel scaffold for $T$ cell memory response. (A) Splenocytes isolated from tumor-bearing control and treated mice were analyzed for the presence of $\mathrm{CD} 8^{+} \mathrm{CD} 44^{+} \mathrm{CD} 122^{+}$ and $\mathrm{CD} 4^{+} \mathrm{CD} 44^{+} \mathrm{CD} 122^{+}$central memory $T$ cells $\left(T_{C M}\right)$. (B and $\left.C\right)$ Corresponding quantification of $\mathrm{CD} 4$ (B) and $\mathrm{CD} 8$ (C) $T_{C M}$ in splenocytes. (D) In vivo bioluminescence imaging of mice after rechallenging with intravenous injection of B16F10 cancer cells. (E) Representative lung photographs (day 10) and (F) H\&E staining of lungs collected from control (naïve) and treated (cured) mice after rechallenging. The blue arrowheads indicate metastatic tumors in the lungs. Scale bar, $100 \mu \mathrm{m}$. (G) Survival curves for naïve and treated mice. Six mice for each groupare shown. Data are means \pm SEM. Statistical significance was calculated by one-way ANOVA with Tukeys post hoc test. ${ }^{* *} P<0.01$.
A
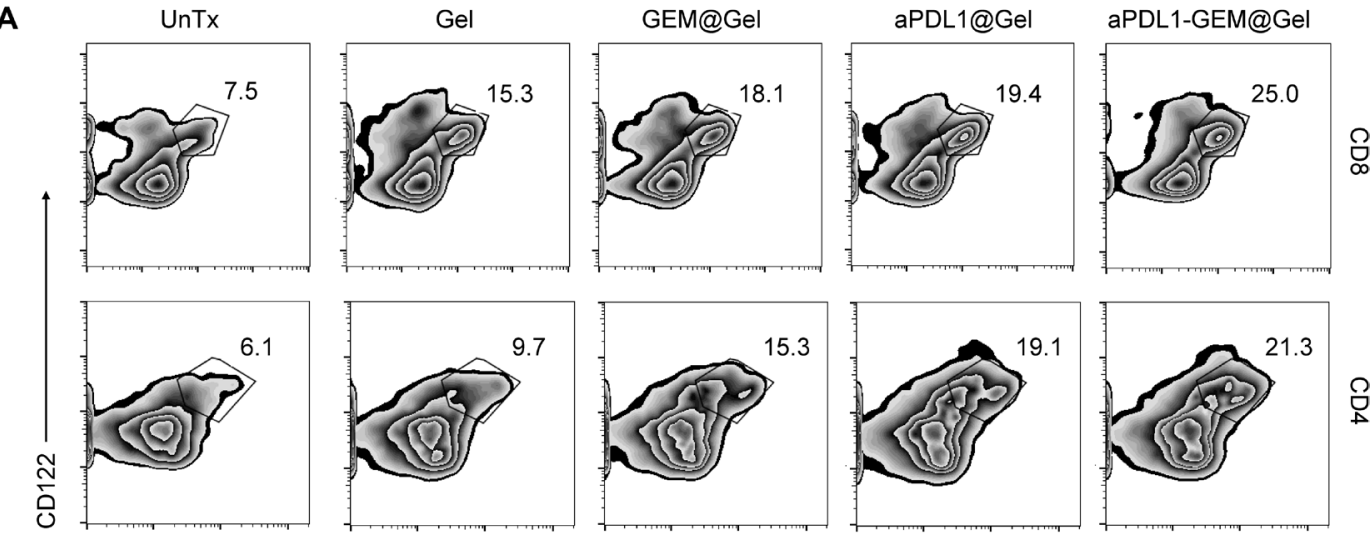

B
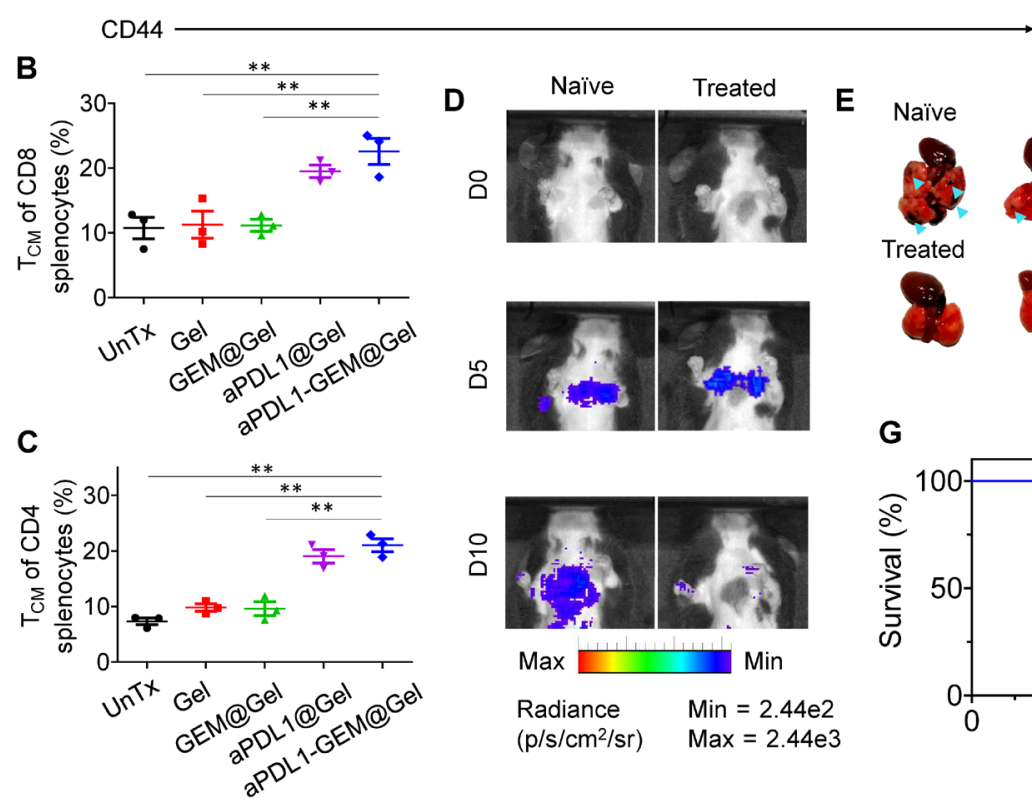

G

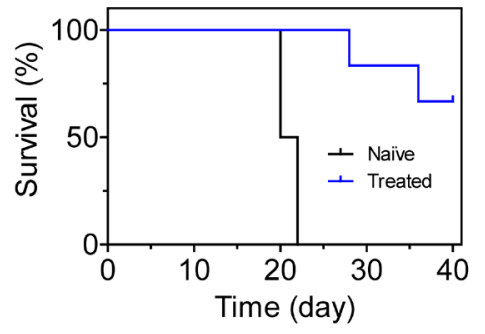


A

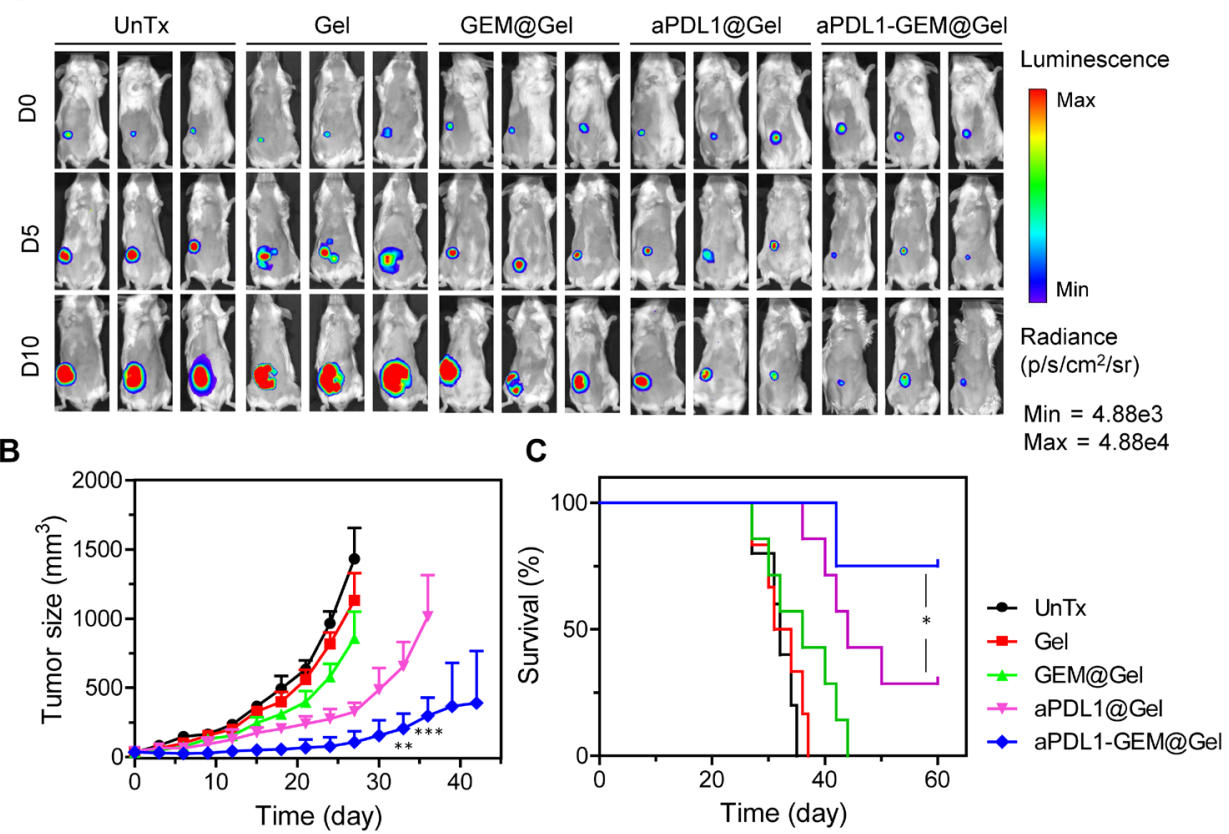

Fig. 6. Local gel scaffold for treatment of low-immunogenic 4T1 carcinoma tumor. (A) In vivo bioluminescence imaging of the 4T1 tumor growth in control and treated mice. (B) Tumor growth kinetics in control and treated mice. Growth curves represent means \pm SEM; growth curves were stopped when the first animal of the corresponding group was euthanized. (C) Survival curves for control and treated mice ( $n=5$ to 10). Statistical significance was calculated by one-way ANOVA with Tukey's post hoc test. ${ }^{*} P<0.05 ;{ }^{* *} P<0.01 ;{ }^{* *} P<0.001$.

of mice did not significantly vary during the treatment (fig. S18). In addition, similar results were obtained in the $4 \mathrm{~T} 1$ tumor recurrence model [we used non-luciferase-expressing 4T1 tumor in this experiment to avoid immune response against the luciferase reporter (55)] (Fig. 8). aPDL1-GEM@Gel treatment prevented cancer recurrence without obvious toxicity (Fig. 8). Collectively, these results suggest that ROS-responsive scaffolds serve as a therapeutic depot to enhance ICB after surgery to inhibit cancer recurrence.

\section{DISCUSSION}

We have demonstrated that an in situ formed hydrogel scaffold consisting of a ROS-sensitive moiety can locally deliver GEM and aPDL1 with distinct kinetics in tumor-bearing mice to promote an immunogenic tumor phenotype and immune-mediated tumor rejection. Previous reports showed that PD-L1 expression in tumor cells was considerably increased after chemotherapy, resulting in PD-L1-mediated T cell exhaustion $(56,57)$. We therefore hypothesized that greater anticancer efficacy could be achieved by local administration of chemotherapeutics and ICB inhibitor with distinct kinetics. To achieve this cascade-like treatment at the tumor site, a ROS-responsive hydrogel scaffold was synthesized and loaded with GEM and aPDL1, taking into account that ROS are abundant in the TME. After in situ construction, the ROS-responsive hydrogel released both GEM and aPDL1 in a ROS-dependent manner. The smaller molecular weight of GEM compared to aPDL1 contributed to its faster release from the hydrogel.

GEM is a ribonucleotide reductase inhibitor that has a broad spectrum of antitumor activity (58). We observed up-regulation of
PD-L1 and PD-1 expression in tumor cells and TILs, respectively, upon exposure to the GEM@Gel.GEM@Gel also reduced tumor-infiltrating MDSCs, which contribute to dysfunction of effector $\mathrm{T}$ cells (37). Because GEM inhibits intratumoral MDSCs (58), their depletion upon the implantation of GEM@Gel was not surprising. Following a similar pattern, GEM@Gel also induced an enhancement of $\mathrm{T}$ cell infiltration and a loss of TAMs expressing CD206. However, loss of TAMs and reduction of ROS were also observed in mice treated with the empty hydrogel. Because ROS is critical for macrophage differentiation, ROS depletion caused by the hydrogel may contribute to recruiting $\mathrm{T}$ cells into the tumor site as well as blocking M2 macrophage differentiation (59). Therefore, the ROS-responsive scaffold may not only act as a reservoir to control the release of therapeutics but also serve as a scavenger of ROS within the TME to enhance treatment efficacy.

aPDL1-GEM@Gel induced an immunogenic tumor phenotype, and the activity of the aPDL1 promoted tumor regression in the $\mathrm{B} 16 \mathrm{~F} 10$ melanoma and $4 \mathrm{~T} 1$ breast tumor mouse models - the latter of which has relatively low PD-L1 expression (38). Moreover, the local treatment generated a systemic anticancer immune response that inhibited distant tumor growth. Collectively, the proposed combined chemoimmunotherapy strategy may offer opportunities for treating poorly immunogenic tumors and reducing systemic toxicities. Furthermore, the scaffold can also be applied to the surgical bed of resected tumors, which is also abundant in ROS (60), making this strategy clinically relevant for inhibiting cancer recurrence postoperatively. Our scaffold generated inside the resection cavity may contribute to scavenging ROS and thus also reduce the inflammation. Finally, although ICB is considered to be tolerated by patients, combination therapy may increase the risk of side effects. Here, we did not observe any obvious toxicity in mice treated with the drug-loaded scaffolds.

Regarding further translation for clinical applications, long-term toxicity of using the scaffold should be thoroughly evaluated. In addition, the dosages of combination drugs as well as treatment frequencies could be further studied and optimized.

In summary, we have developed a combined chemoimmunotherapy strategy based on the controlled release of chemotherapeutic drug and ICB inhibitor from a TME-responsive hydrogel scaffold. GEM released by the hydrogel scaffold elicits immunogenic phenotypes in tumors. Notably, the ROS-responsive gels can not only serve as a reservoir for tuning release of therapeutics but also function as a scavenger of ROS and thus further enhance the immunogenic phenotypes. This strategy holds promise for treating low-immunogenic tumors that are poorly responsive to ICB. Moreover, because the local implantation of the scaffold promotes systemic immune 


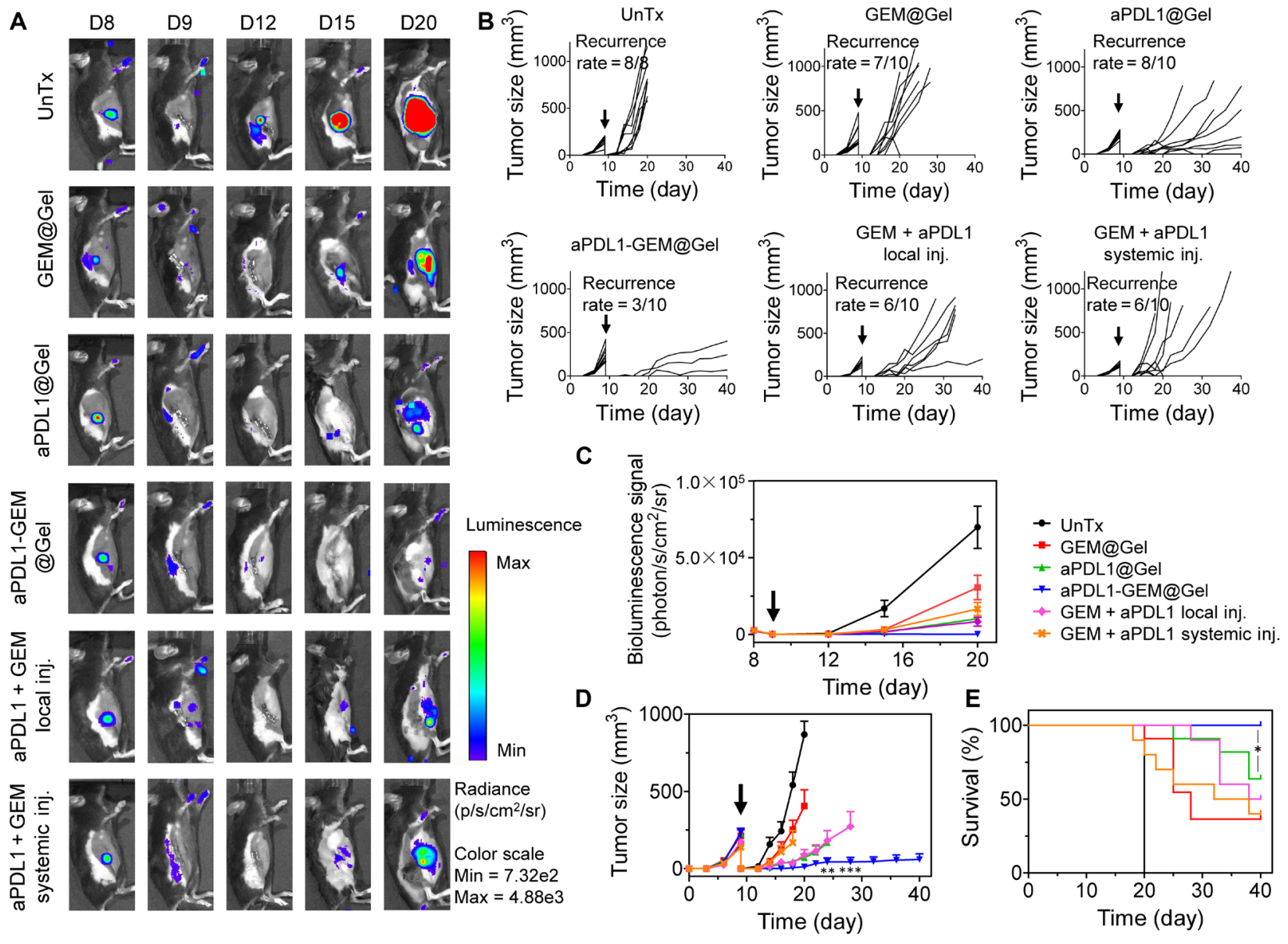

Fig. 7. Gel scaffold for inhibition of postsurgical recurrence of B16F10 tumors. (A) In vivo bioluminescence imaging of the B16F10 tumor growth in C57B6 mice after various treatments as indicated. (B to D) Individual (B) and average quantitative bioluminescence signals of tumors (C) and tumor growth kinetics (D) in control and treated groups. Black arrows indicate the day of the surgery (day 9). (E) Survival curves for different treatments ( $n=7$ to 10$)$. Growth curves were stopped when the first animal of the corresponding group was euthanized. Data are means \pm SEM. Statistical significance was calculated by one-way ANOVA with Tukey's post hoc test. * $P<0.05$; ${ }^{* *} P<0.01 ;{ }^{* * *} P<0.001$.

Fig. 8. Gel scaffold for inhibition of postsurgical recurrence of $4 \mathrm{~T} 1$ tumors. (A) Individual and (B) average tumor growth kinetics in control and treated groups receiving the indicated treatments. Growth curves were stopped when the first animal of the corresponding group was euthanized ordead. (C) Survival curves for different treatments ( $n=5$ to 9 , as indicated in the figure). (D) Measurements of body weight of control and treated mice. Black arrows indicate the day of the surgery (day 14). Data are means \pm SEM. Statistical significance was calculated by one-way ANOVA with Tukey's post hoc test. ${ }^{*} P<0.05$.
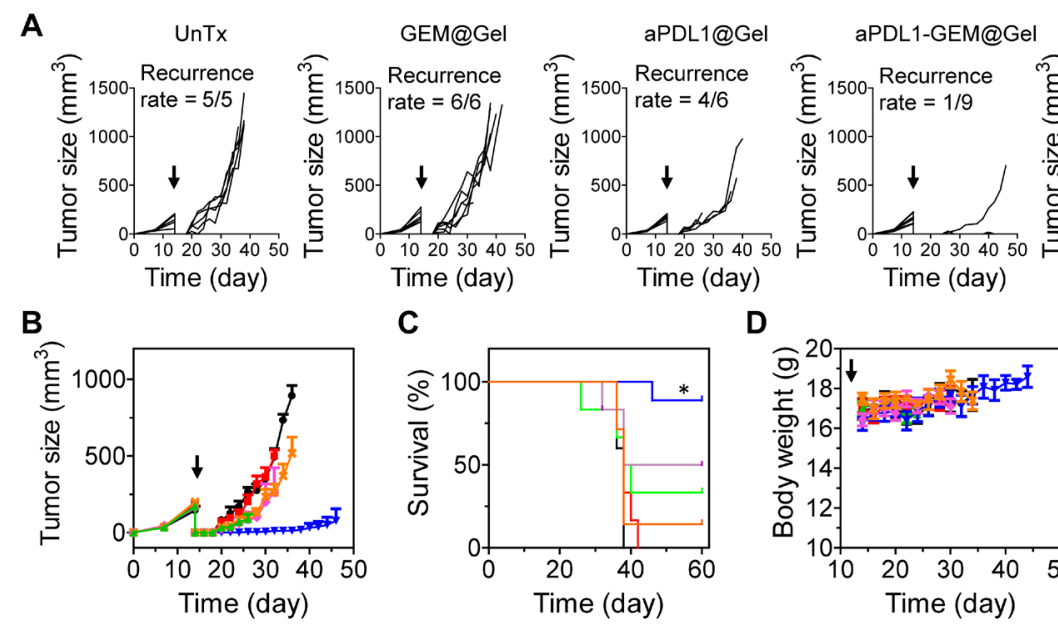

C

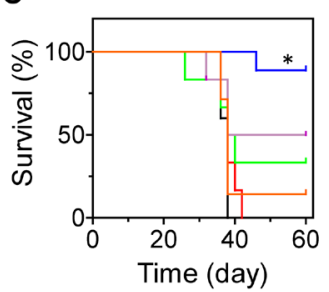

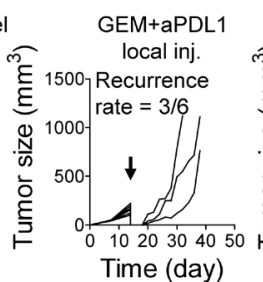

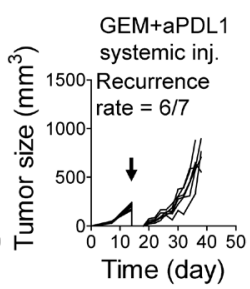

D

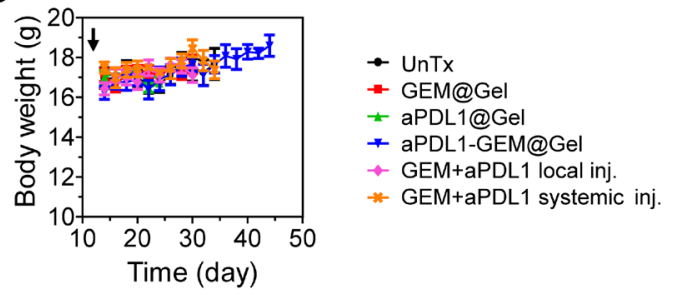


responses and $\mathrm{T}$ cell memory formation, this strategy may be useful to treat metastatic tumors and inhibit tumor recurrence.

\section{MATERIALS AND METHODS Study design}

The objective of the study was to develop a strategy based on controlled release of chemotherapeutic drug and ICB from a TMEresponsive hydrogel scaffold for enhancing response and efficiency of ICB therapy. The in vivo antitumor efficacy was assessed in B16F10 and 4T1 tumors and incomplete tumor resection models. Mice from varying treatment groups were followed to create survival curves, imaged to assess tumor progression, and rechallenged with tumor to assess immune memory. Sample sizes were determined on the basis of our previous experimental experience. Animals were randomly assigned to groups based on tumor size and body weight. The investigators were not blinded to allocation during experiments and outcome assessment. Animals were euthanized when exhibiting signs of impaired health or when the volume of the tumor exceeded $1.5 \mathrm{~cm}^{3}$. All experiments were run at least in triplicate.

\section{Materials}

All chemicals were purchased from Sigma-Aldrich unless otherwise specified and were used as received. GEM hydrochloride (United States Pharmacopeia reference standard) was purchased from Sigma (catalog no. 1288463). aPDL1 used in vivo was purchased from BioLegend Inc. (catalog no. 124329, clone 10F.9G2).

\section{Cell lines}

The mouse melanoma cell line B16F10 and mouse mammary carcinoma cell line 4T1 were purchased from the American Type Culture Collection. B16F10-luc-GFP and 4T1-luc-GFP cells were gifts from L. Huang at the University of North Carolina at Chapel Hill (UNC-CH). B16F10 cells were maintained in Dulbecco's modified Eagle's medium (Gibco, Invitrogen) supplemented with $10 \%$ fetal bovine serum (Invitrogen), penicillin $(100 \mathrm{U} / \mathrm{ml}$; Invitrogen), and streptomycin (100 U/ml; Invitrogen). 4T1 cells were maintained in RPMI 1640 medium (Gibco, Invitrogen) supplemented with $10 \%$ fetal bovine serum (Invitrogen), penicillin (100 U/ml; Invitrogen), and streptomycin (100 U/ml; Invitrogen). Master and working cell banks were generated immediately upon receipt. The third and fourth passages were used for the experiments. Cells were tested every 3 months to exclude the presence of mycoplasma. Authentication of cells was not performed after receipt.

\section{Mice}

C57BL/ 6 and BALB/c mice were purchased from The Jackson Laboratory. Age-matched (6 to 10 weeks) female mice were used throughout all experiments. We performed all mouse studies in accordance with the animal protocol approved by the Institutional Animal Care and Use Committee at UNC-CH and North Carolina State University (NCSU). Experimental group sizes were approved by the regulatory authorities for animal welfare after being defined to balance statistical power, feasibility, and ethical aspects. All mice were kept in accordance with federal and state policies on animal research at UNC-CH and NCSU.

\section{Antibodies}

aPDL1 used in vivo was purchased from BioLegend Inc. (catalog no. 124329, clone 10F.9G2). Antibodies used for flow cytometry included CD3 (catalog no. A18644, Thermo Fisher Scientific), CD4 (catalog no. A18667, Thermo Fisher Scientific), CD8 (catalog no. A18609, Thermo Fisher Scientific), PD-1 (catalog no. 135227, BioLegend), CD11c (catalog no. 117309, BioLegend), PD-L1 (catalog no. 124311, BioLegend), CD11b (catalog no. 101211, BioLegend), and intracellular FOXP3 (catalog no. 71-5775-40, eBioscience). The stained cells were analyzed on a CytoFLEX flow cytometer (Beckman Coulter). A minimum of 1000 events per plot were collected and analyzed using FlowJo software (version 10). Secondary antibodies including goat anti-rat IgG $(\mathrm{H}+\mathrm{L})$ (catalog no. A18866, Thermo Fisher Scientific), rabbit anti-rat $\operatorname{IgG}(\mathrm{H}+\mathrm{L})$ (catalog no. A18920, Thermo Fisher Scientific), and goat anti-rat IgG (minimal x-reactivity) (catalog no. 405408, BioLegend) were used for immunostaining.

\section{Synthesis of TSPBA}

$N, N, N^{\prime}, N^{\prime}$-tetramethyl-1,3-propanediamine $(0.1 \mathrm{~g}, 0.75 \mathrm{mmol})$ and 4-(bromomethyl)phenylboronic acid ( $0.5 \mathrm{~g}, 2.3 \mathrm{mmol})$ were dissolved in dimethylformamide $(10 \mathrm{ml})$ and mixed together. After stirring at $60^{\circ} \mathrm{C}$ overnight, the mixture was poured into tetrahydrofuran (THF) $(100 \mathrm{ml})$, filtered, and washed with THF $(3 \times 20 \mathrm{ml})$. After drying under vacuum overnight, pure TSPBA $(0.3 \mathrm{~g}$, yield $70 \%)$ was obtained. ${ }^{1} \mathrm{H}$ nuclear magnetic resonance $(300 \mathrm{MHz}$, d-DMSO, $\delta): 8.132$ (s, 4H), $7.85(\mathrm{~d}, 4 \mathrm{H}), 7.49$ (d, $4 \mathrm{H}), 4.58(\mathrm{~s}, 4 \mathrm{H})$, $3.26(\mathrm{~s}, 4 \mathrm{H}), 2.97$ (s, 12H), $2.38(\mathrm{~m}, 2 \mathrm{H})$ (fig. S2).

\section{Formation of PVA-TSPBA hydrogels}

PVA (72 kDa; 98\% hydrolyzed; $5 \mathrm{~g}$ ) and deionized water $(100 \mathrm{ml})$ were mixed together and stirred at $90^{\circ} \mathrm{C}$ to acquire a clear solution. TSPBA [ 5 weight \% (wt \%) in $\mathrm{H}_{2} \mathrm{O}, 2 \mathrm{ml}$ ] and PVA ( 5 wt $\%$ in $\mathrm{H}_{2} \mathrm{O}$, $2 \mathrm{ml}$ ) were mixed together, and a hydrogel was formed instantly. This hydrogel was used for in vitro experiments. For the fabrication of GEM- and aPDL1-loaded gel, predetermined amount of GEM or aPDL1 was added to the PVA aqueous solution. For in vivo application, PVA and TSPBA aqueous solutions were loaded into dual syringes and injected directly toward tumors to form a gel in situ.

\section{Characterization of aPDL1-GEM@Gel}

Cryo-SEM imaging was obtained by JEOL 7600F with Gatan Alto. Fluorescence imaging was analyzed using a confocal microscope (Zeiss LSM 710). The dynamic rheological behavior of PVA before and after gelation at $25^{\circ} \mathrm{C}$ was measured using a TA Instruments AR 2000 stress controlled rheometer with 25 -mm aluminum crosshatched parallel plates.

\section{GEM and aPDL1 release from PVA-TSPBA hydrogels}

Release studies were performed at $37^{\circ} \mathrm{C}$ with constant agitation in PBS. $\mathrm{H}_{2} \mathrm{O}_{2}$ (Sigma) was added to samples to study the GEM and antibody release. The released GEM was analyzed by HPLC, and the antibody release was determined by the Rat IgG Total ELISA Kit (eBioscience).

\section{In vivo tumor models}

To test the anticancer effects in mouse models, mice were studied 9 days after $1 \times 10^{6}$ of luciferase-tagged B16F10 or 14 days after $1 \times 10^{6}$ 
of luciferase-tagged $4 \mathrm{~T} 1$ tumor cells were transplanted into the right flank of mice. Mice were weighed and randomly divided into different groups ( $n=7$ to 10 ). The mice were peritumorally implanted with different formulations, including hydrogels, GEM@ Gel, aPDL1@Gel, and aPDL1-GEM@Gel (aPDL1, 50 pg per mouse; GEM, $5 \mathrm{mg} / \mathrm{kg} ; 200 \mu \mathrm{l} ; 10 \%$, w/w). The tumor burden was monitored by the bioluminescence signal of cancer cells. Images were taken using an IVIS Lumina imaging system (Caliper). The tumors were also measured with a digital caliper. The tumor volume $\left(\mathrm{mm}^{3}\right)$ was calculated as (long diameter $\times$ short diameter ${ }^{2}$ )/2.

To measure the effects on cancer recurrence, 9 days after $1 \times 10^{6}$ of B16F10/luciferase-tagged B16F10 or 14 days after $1 \times 10^{6}$ of $4 \mathrm{~T} 1 /$ luciferase-tagged $4 \mathrm{~T} 1$ tumor cells were transplanted into the right flank of mice, the tumors were resected, leaving about $1 \%$ residual tissue behind to mimic the residual microtumors in a surgical bed $(53,54)$. Briefly, animals were anesthetized with isoflurane (1 to $3 \%$ for maintenance; up to $5 \%$ for induction) anesthesia via chamber induction and maintained via nose cone. The amount of residual tumor tissue was determined by the bioluminescence signals of tumor cells before and after resection. The tumor area was clipped and aseptically prepared. Sterile instruments were used to remove roughly $99 \%$ of the tumor. The amount of residual tumor tissue was determined by the integrated bioluminescence signal intensity of the tumor tissues before and after tumor resection. The wound was closed by Autoclip wound clip system. Mice were weighed and randomly divided into different groups ( $n=5$ to 10$)$. After surgery, different formulations were implanted into the surgical bed, including hydrogels,GEM@Gel, aPDL1@Gel, and aPDL1-GEM@Gel. Free GEM + aPDL1 at the same dose were locally or systemically administered into mice after resection of primary tumors. The tumor burden was monitored by the bioluminescence signal of cancer cells. The mice were clipped and shaved using a depilatory cream before imaging, if necessary. The tumors were also measured with a digital caliper. The tumor volume $\left(\mathrm{mm}^{3}\right)$ was calculated as (long diameter $\times$ short diameter $\left.{ }^{2}\right) / 2$. Animals were euthanized with carbon dioxide when exhibiting signs of impaired health or when the volume of the tumor exceeded $1.5 \mathrm{~cm}^{3}$.

\section{In vivo bioluminescence and imaging}

Bioluminescence images were collected with the IVIS Spectrum Imaging System (PerkinElmer Ltd.). Living Image software (PerkinElmer Ltd.) was used to acquire the data $10 \mathrm{~min}$ after intraperitoneal injection of D-luciferin (Thermo Scientific Pierce) in Dulbecco's PBS (15 mg/ml) into the animals (body weight, $10 \mu \mathrm{l} / \mathrm{g}$ ). Exposure time for bioluminescence imaging was $5 \mathrm{~min}$. Regions of interest were quantified as average radiance (photons s $\mathrm{sm}^{-1} \mathrm{sr}^{-1}$, represented by color bars) (IVIS Living Image 4.2).

\section{Cytokine detection}

The plasma concentrations of IL-2, IL-6, IL-10, IFN- $\gamma$, and TNF- $\alpha$ (tumor necrosis factor- $\alpha$ ) were measured by LEGENDplex Mouse Th1 Panel multiple assay (catalog no. 740025, BioLegend) according to the manufacturer's instructions. The plasma was collected from mice before and 2 days after GEM@Gel implantation.

\section{Confocal microscopy}

Harvested tumors were dissected and snap-frozen in optimal cutting temperature compound (Thermo Fisher Scientific). Micrometer sections were cut using a cryotome and mounted on slides. Sections were fixed in ice-cold acetone for $10 \mathrm{~min}$ before rehydration with PBS. After blocking with bovine serum albumin (3\%), sections were stained with primary antibodies overnight at $4^{\circ} \mathrm{C}$. After the addition of fluorescence-labeled secondary antibodies, the slides were analyzed using a confocal microscope (Zeiss LSM 710).

\section{Statistical analysis}

All results are expressed as means \pm SD or means \pm SEM, as indicated. Biological replicates were used in all experiments unless stated otherwise. ANOVA was performed when more than two groups were compared, and when the result was significant $(P<0.05)$, multiple comparisons were performed using Tukey's post hoc test. Statistical differences in survival were determined with the log-rank test. All statistical analyses were performed with GraphPad Prism (5.0). ${ }^{*} P<$ $0.05,{ }^{* *} P<0.01,{ }^{* *} P<0.005$.

\section{SUPPLEMENTARY MATERIALS}

www.sciencetranslationalmedicine.org/cgi/content/full/10/429/eaan3682/DC1 Materials and Methods

Fig. S1. Schematic of the $\mathrm{H}_{2} \mathrm{O}_{2}$ responsiveness mechanism of the PVA-TSPBA gel.

Fig. S2. Synthesis route and characterization of TSPBA.

Fig. S3. Dynamic rheological behavior of PVA before and after gelation.

Fig. S4. In vivo gel maintenance.

Fig. S5. Oxidation and hydrolysis of TSPBA.

Fig. S6. In vivo release of payloads from gel scaffold.

Fig. S7. Low-dose GEM@Gel for enhancement of lymphocyte infiltration.

Fig. S8. ROS-responsive scaffold as a scavenger of ROS within the TME.

Fig. S9. Frequency of $C D 4^{+} \mathrm{FOXP}^{+} \mathrm{T}$ cells within the tumors of mice receiving the indicated treatments.

Fig. S10. Effects of GEM on cancer cells in vitro.

Fig. S11. In vivo PD-L1 expression in tumor cells at different time points.

Fig. S12. Effects of GEM@Gel on systemic concentrations of cytokines.

Fig. S13. Tumor inhibition in mice treated with free drugs compared to aPDL1-GEM@Gel.

Fig. S14. Characterization of T cell-mediated antitumor immune response.

Fig. S15. PD-L1 expression in distant tumors.

Fig. S16. Effects of GEM on 4T1 cells in vitro.

Fig. S17. Biocompatibility of hydrogel in vivo.

Fig. S18. Body weight of control and treated mice.

\section{REFERENCES AND NOTES}

1. W. Zou, J. D. Wolchok, L. Chen, PD-L1 (B7-H1) and PD-1 pathway blockade for cancer therapy: Mechanisms, response biomarkers, and combinations. Sci. Transl. Med. 8, 328rv4 (2016).

2. V. A. Boussiotis, Molecular and biochemical aspects of the PD-1 checkpoint pathway. N. Engl. J. Med. 375, 1767-1778 (2016).

3. D. R. Littman, Releasing the brakes on cancer immunotherapy. Cell 162, 1186-1190 (2015).

4. L. E. Klevorn, R. M. Teague, Adapting cancer immunotherapy models for the real world. Trends Immunol. 37, 354-363 (2016).

5. J. E. Rosenberg, J. Hoffman-Censits, T. Powles, M. S. van der Heijden, A. V. Balar, A. Necchi, N. Dawson, P. H. O'Donnell, A. Balmanoukian, Y. Loriot, S. Srinivas, M. M. Retz, P. Grivas, R. W. Joseph, M. D. Galsky, M. T. Fleming, D. P. Petrylak, J. L. Perez-Gracia, H. A. Burris, D. Castellano, C. Canil, J. Bellmunt, D. Bajorin, D. Nickles, R. Bourgon, G. M. Frampton, N. Cui, S. Mariathasan, O. Abidoye, G. D. Fine, R. Dreicer, Atezolizumab in patients with locally advanced and metastatic urothelial carcinoma who have progressed following treatment with platinum-based chemotherapy: A single-arm, multicentre, phase 2 trial. Lancet 387, 1909-1920 (2016).

6. E. I. Buchbinder, F. S. Hodi, Melanoma in 2015: Immune-checkpoint blockade-Durable cancer control. Nat. Rev. Clin. Oncol. 13, 77-78 (2016).

7. D. Killock, Lung cancer: Anti-PD-1 therapy in the frontline. Nat. Rev. Clin. Oncol. 13, 715 (2016).

8. T. N. Schumacher, R. D. Schreiber, Neoantigens in cancer immunotherapy. Science $\mathbf{3 4 8}$, 69-74 (2015).

9. H. Tang, Y. Wang, L. K. Chlewicki, Y. Zhang, J. Guo, W. Liang, J. Wang, X. Wang, Y.-X. Fu, Facilitating $T$ cell infiltration in tumor microenvironment overcomes resistance to PD-L1 blockade. Cancer Cell 30, 500 (2016). 
10. S. L. Topalian, J. M. Taube, R. A. Anders, D. M. Pardoll, Mechanism-driven biomarkers to guide immune checkpoint blockade in cancer therapy. Nat. Rev. Cancer 16, 275-287 (2016).

11. G. T. Gibney, L. M. Weiner, M. B. Atkins, Predictive biomarkers for checkpoint inhibitorbased immunotherapy. Lancet Oncol. 17, e542-e551 (2016).

12. P. Sharma, J. P. Allison, The future of immune checkpoint therapy. Science 348, 56-61 (2015).

13. P. Sharma, J. P. Allison, Immune checkpoint targeting in cancer therapy: Toward combination strategies with curative potential. Cell 161, 205-214 (2015).

14. C. Boutros, A. Tarhini, E. Routier, O. Lambotte, F. L. Ladurie, F. Carbonnel, H. Izzeddine, A. Marabelle, S. Champiat, A. Berdelou, E. Lanoy, M. Texier, C. Libenciuc, A. M. M. Eggermont, J.-C. Soria, C. Mateus, C. Robert, Safety profiles of anti-CTLA-4 and anti-PD-1 antibodies alone and in combination. Nat. Rev. Clin. Oncol. 13, 473-486 (2016).

15. J. Naidoo, D. B. Page, B. T. Li, L. C. Connell, K. Schindler, M. E. Lacouture, M. A. Postow, J. D. Wolchok, Toxicities of the anti-PD-1 and anti-PD-L1 immune checkpoint antibodies. Ann. Oncol. 26, 2375-2391 (2015).

16. D. B. Johnson, J. M. Balko, M. L. Compton, S. Chalkias, J. Gorham, Y. Xu, M. Hicks, I. Puzanov, M. R. Alexander, T. L. Bloomer, J. R. Becker, D. A. Slosky, E. J. Phillips, M. A. Pilkinton, L. Craig-Owens, N. Kola, G. Plautz, D. S. Reshef, J. S. Deutsch, R. P. Deering, B. A. Olenchock, A. H. Lichtman, D. M. Roden, C. E. Seidman, I. J. Koralnik, J. G. Seidman, R. D. Hoffman, J. M. Taube, L. A. Diaz Jr., R. A. Anders, J. A. Sosman, J. J. Moslehi, Fulminant myocarditis with combination immune checkpoint blockade. N. Engl. J. Med. 375, 1749-1755 (2016).

17. K. D. Moynihan, C. F. Opel, G. L. Szeto, A. Tzeng, E. F. Zhu, J. M. Engreitz, R. T. Williams, K. Rakhra, M. H. Zhang, A. M. Rothschilds, S. Kumari, R. L. Kelly, B. H. Kwan, W. Abraham, K. Hu, N. K. Mehta, M. J. Kauke, H. Suh, J. R. Cochran, D. A. Lauffenburger, K. D. Wittrup, D. J. Irvine, Eradication of large established tumors in mice by combination immunotherapy that engages innate and adaptive immune responses. Nat. Med. 22, 1402-1410 (2016).

18. E. Vacchelli, Y. Ma, E. E. Baracco, A. Sistigu, D. P. Enot, F. Pietrocola, H. Yang, S. Adjemian, K. Chaba, M. Semeraro, M. Signore, A. De Ninno, V. Lucarini, F. Peschiaroli, L. Businaro, A. Gerardino, G. Manic, T. Ulas, P. Günther, J. L. Schultze, O. Kepp, G. Stoll, C. Lefebvre, C. Mulot, F. Castoldi, S. Rusakiewicz, S. Ladoire, L. Apetoh, J. M. Bravo-San Pedro, M. Lucattelli, C. Delarasse, V. Boige, M. Ducreux, S. Delaloge, C. Borg, F. André, G. Schiavoni, I. Vitale, P. Laurent-Puig, F. Mattei, L. Zitvogel, G. Kroemer, Chemotherapyinduced antitumor immunity requires formyl peptide receptor 1. Science 350, 972-978 (2015).

19. S. Böhm, A. Montfort, O. M. T. Pearce, J. Topping, P. Chakravarty, G. L. A. Everitt, A. Clear, J. R. McDermott, D. Ennis, T. Dowe, A. Fitzpatrick, E. C. Brockbank, A. C. Lawrence, A. Jeyarajah, A. Z. Faruqi, I. A. McNeish, N. Singh, M. Lockley, F. R. Balkwill, Neoadjuvant chemotherapy modulates the immune microenvironment in metastases of tubo-ovarian high-grade serous carcinoma. Clin. Cancer Res. 22, 3025-3036 (2016).

20. C. Pfirschke, C. Engblom, S. Rickelt, V. Cortez-Retamozo, C. Garris, F. Pucci, T. Yamazaki, V. Poirier-Colame, A. Newton, Y. Redouane, Y.-J. Lin, G. Wojtkiewicz, Y. Iwamoto, M. Mino-Kenudson, T. G. Huynh, R. O. Hynes, G. J. Freeman, G. Kroemer, L. Zitvogel, R. Weissleder, M. J. Pittet, Immunogenic chemotherapy sensitizes tumors to checkpoint blockade therapy. Immunity 44, 343-354 (2016).

21. D. Mathios, J. E. Kim, A. Mangraviti, J. Phallen, C.-K. Park, C. M. Jackson, T. Garzon-Muvdi, E. Kim, D. Theodros, M. Polanczyk, A. M. Martin, I. Suk, X. Ye, B. Tyler, C. Bettegowda, H. Brem, D. M. Pardoll, M. Lim, Anti-PD-1 antitumor immunity is enhanced by local and abrogated by systemic chemotherapy in GBM. Sci. Transl. Med. 8, 370ra180 (2016).

22. E. Nolan, P. Savas, A. N. Policheni, P. K. Darcy, F. Vaillant, C. P. Mintoff, S. Dushyanthen, M. Mansour, J.-M. B. Pang, S. B. Fox; Kathleen Cuningham Foundation Consortium for Research into Familial Breast Cancer (kConFab), C. M. Perou, J. E. Visvader, D. H. D. Gray, S. Loi, G. J. Lindeman, Combined immune checkpoint blockade as a therapeutic strategy for BRCA1-mutated breast cancer. Sci. Transl. Med. 9, eaal4922 (2017).

23. M. Black, I. B. Barsoum, P. Truesdell, T. Cotechini, S. K. Macdonald-Goodfellow, M. Petroff, D. R. Siemens, M. Koti, A. W. B. Craig, C. H. Graham, Activation of the PD-1/PD-L1 immune checkpoint confers tumor cell chemoresistance associated with increased metastasis. Oncotarget 7, 10557-10567 (2016).

24. L. Galluzzi, A. Buqué, O. Kepp, L. Zitvogel, G. Kroemer, Immunogenic cell death in cancer and infectious disease. Nat. Rev. Immunol. 17, 97-111 (2017).

25. A. M. Cook, W. J. Lesterhuis, A. K. Nowak, R. A. Lake, Chemotherapy and immunotherapy: Mapping the road ahead. Curr. Opin. Immunol. 39, 23-29 (2016).

26. E. P.-Y. Lin, C.-Y. Yang, C.-W. Lin, B.-T. Huang, W.-Y. Lai, Y.-T. Tseng, P.-C. Yang, Priming PD-L1 expression by chemotherapeutic agents in non-small cell lung cancers. J. Clin. Oncol. 35, e20087 (2017).

27. L. Gu, D. J. Mooney, Biomaterials and emerging anticancer therapeutics: Engineering the microenvironment. Nat. Rev. Cancer 16, 56-66 (2016).

28. N. A. Hotaling, L. Tang, D. J. Irvine, J. E. Babensee, Biomaterial strategies for immunomodulation. Annu. Rev. Biomed. Eng. 17, 317-349 (2015).

29. A. M. Rosales, K. S. Anseth, The design of reversible hydrogels to capture extracellular matrix dynamics. Nat. Rev. Mater. 1, 15012 (2016).
30. C. Wang, Y. Ye, G. M. Hochu, H. Sadeghifar, Z. Gu, Enhanced cancer immunotherapy by microneedle patch-assisted delivery of anti-PD1 antibody. Nano Lett. 16, 2334-2340 (2016).

31. A. J. Vegas, O. Veiseh, J. C. Doloff, M. Ma, H. H. Tam, K. Bratlie, J. Li, A. R. Bader, E. Langan, K. Olejnik, P. Fenton, J. W. Kang, J. Hollister-Locke, M. A. Bochenek, A. Chiu, S. Siebert, K. Tang, S. Jhunjhunwala, S. Aresta-Dasilva, N. Dholakia, R. Thakrar, T. Vietti, M. Chen, J. Cohen, K. Siniakowicz, M. Qi, J. McGarrigle, A. C. Graham, S. Lyle, D. M. Harlan, D. L. Greiner, J. Oberholzer, G. C. Weir, R. Langer, D. G. Anderson, Combinatorial hydrogel library enables identification of materials that mitigate the foreign body response in primates. Nat. Biotechnol. 34, 345-352 (2016).

32. N. Reznikov, J. A. M. Steele, P. Fratzl, M. M. Stevens, A materials science vision of extracellular matrix mineralization. Nat. Rev. Mater. 1, 16041 (2016).

33. C. Wang, Y. Ye, Q. Hu, A. Bellotti, Z. Gu, Tailoring biomaterials for cancer immunotherapy: Emerging trends and future outlook. Adv. Mater. 29, 1606036 (2017).

34. G.-Y. Liou, P. Storz, Reactive oxygen species in cancer. Free Radic. Res. 44, 479-496 (2010).

35. C. Nathan, A. Cunningham-Bussel, Beyond oxidative stress: An immunologist's guide to reactive oxygen species. Nat. Rev. Immunol. 13, 349-361 (2013).

36. N. S. Katheder, R. Khezri, F. O'Farrell, S. W. Schultz, A. Jain, M. M. Rahman, K. O. Schink, T. A. Theodossiou, T. Johansen, G. Juhász, D. Bilder, A. Brech, H. Stenmark, T. E. Rusten, Microenvironmental autophagy promotes tumour growth. Nature 541, 417-420 (2017).

37. D. I. Gabrilovich, S. Nagaraj, Myeloid-derived suppressor cells as regulators of the immune system. Nat. Rev. Immunol. 9, 162-174 (2009).

38. I. Sagiv-Barfi, H. E. K. Kohrt, D. K. Czerwinski, P. P. Ng, B. Y. Chang, R. Levy, Therapeutic antitumor immunity by checkpoint blockade is enhanced by ibrutinib, an inhibitor of both BTK and ITK. Proc. Natl. Acad. Sci. U.S.A. 112, E966-E972 (2015).

39. T. Konno, K. Ishihara, Temporal and spatially controllable cell encapsulation using a water-soluble phospholipid polymer with phenylboronic acid moiety. Biomaterials $\mathbf{2 8}$, 1770-1777 (2007).

40. S. Kitano, K. Kataoka, Y. Koyama, T. Okano, Y. Sakurai, Glucose-responsive complex formation between poly(vinyl alcohol) and poly( $\mathrm{N}$-vinyl-2-pyrrolidone) with pendent phenylboronic acid moieties. Macromol. Rapid Commun. 12, 227-233 (1991).

41. J. Park, S. H. Wrzesinski, E. Stern, M. Look, J. Criscione, R. Ragheb, S. M. Jay, S. L. Demento, A. Agawu, P. L. Limon, A. F. Ferrandino, D. Gonzalez, A. Habermann, R. A. Flavell, T. M. Fahmy, Combination delivery of TGF- $\beta$ inhibitor and IL-2 by nanoscale liposomal polymeric gels enhances tumour immunotherapy. Nat. Mater. 11, 895-905 (2012).

42. S. P. Arlauckas, C. S. Garris, R. H. Kohler, M. Kitaoka, M. F. Cuccarese, K. S. Yang, M. A. Miller, J. C. Carlson, G. J. Freeman, R. M. Anthony, R. Weissleder, M. J. Pittet, In vivo imaging reveals a tumor-associated macrophage-mediated resistance pathway in anti-PD-1 therapy. Sci. Transl. Med. 9, eaal3604 (2017).

43. M. A. Kursunel, G. Esendagli, The untold story of IFN- $\gamma$ in cancer biology. Cytokine Growth Factor Rev. 31, 73-81 (2016).

44. K. Abiko, N. Matsumura, J. Hamanishi, N. Horikawa, R. Murakami, K. Yamaguchi, Y. Yoshioka, T. Baba, I. Konishi, M. Mandai, IFN- $\gamma$ from lymphocytes induces PD-L1 expression and promotes progression of ovarian cancer. Br. J. Cancer 112, 1501-1509 (2015).

45. M. Mandai, J. Hamanishi, K. Abiko, N. Matsumura, T. Baba, I. Konishi, Dual faces of IFN $\gamma$ in cancer progression: A role of PD-L1 induction in the determination of pro- and antitumor immunity. Clin. Cancer Res. 22, 2329-2334 (2016).

46. R. A. Seder, P. A. Darrah, M. Roederer, T-cell quality in memory and protection: Implications for vaccine design. Nat. Rev. Immunol. 8, 247-258 (2008).

47. L. Z. Shi, T. Fu, B. Guan, J. Chen, J. M. Blando, J. P. Allison, L. Xiong, S. K. Subudhi, J. Gao, P. Sharma, Interdependent IL-7 and IFN- $\gamma$ signalling in T-cell controls tumour eradication by combined $\alpha$-CTLA-4+ $\alpha$-PD-1 therapy. Nat. Commun. 7, 12335 (2016).

48. T. Walzer, C. Arpin, L. Beloeil, J. Marvel, Differential in vivo persistence of two subsets of memory phenotype CD8 T cells defined by CD44 and CD122 expression levels. J. Immunol. 168, 2704-2711 (2002).

49. J. A. Olson, C. McDonald-Hyman, S. C. Jameson, S. E. Hamilton, Effector-like CD8 ${ }^{+}$T cells in the memory population mediate potent protective immunity. Immunity 38, 1250-1260 (2013).

50. J. Liu, D. Chen, G. D. Nie, Z. Dai, $C D 8^{+} \mathrm{CD} 122^{+}$T-cells: A newly emerging regulator with central memory cell phenotypes. Front. Immunol. 6, 494 (2015).

51. T. Yamaoka, Y. Tabata, Y. Ikada, Comparison of body distribution of poly(vinyl alcohol) with other water-soluble polymers after intravenous administration. J. Pharm. Pharmacol. 47, 479-486 (1995).

52. Y. Kaneo, S. Hashihama, A. Kakinoki, T. Tanaka, T. Nakano, Y. Ikeda, Pharmacokinetics and biodisposition of poly(vinyl alcohol) in rats and mice. Drug Metab. Pharmacokinet. 20, 435-442 (2005).

53. C. Wang, W. Sun, Y. Ye, Q. Hu, H. N. Bomba, Z. Gu, In situ activation of platelets with checkpoint inhibitors for post-surgical cancer immunotherapy. Nat. Biomed. Eng. 1, 0011 (2017). 
54. S. B. Stephan, A. M. Taber, I. Jileaeva, E. P. Pegues, C. L. Sentman, M. T. Stephan, Biopolymer implants enhance the efficacy of adoptive T-cell therapy. Nat. Biotechnol. 33, 97-101 (2015).

55. J. C. Tiffen, C. G. Bailey, C. Ng, J. E. J. Rasko, J. Holst, Luciferase expression and bioluminescence does not affect tumor cell growth in vitro or in vivo. Mol. Cancer 9, 299 (2010).

56. P. Zhang, D.-M. Su, M. Liang, J. Fu, Chemopreventive agents induce programmed death-1-ligand 1 (PD-L1) surface expression in breast cancer cells and promote PD-L1-mediated T cell apoptosis. Mol. Immunol. 45, 1470-1476 (2008).

57. N. McGranahan, A. J. S. Furness, R. Rosenthal, S. Ramskov, R. Lyngaa, S. K. Saini, M. Jamal-Hanjani, G. A. Wilson, N. J. Birkbak, C. T. Hiley, T. B. K. Watkins, S. Shafi, N. Murugaesu, R. Mitter, A. U. Akarca, J. Linares, T. Marafioti, J. Y. Henry, E. M. Van Allen, D. Miao, B. Schilling, D. Schadendorf, L. A. Garraway, V. Makarov, N. A. Rizvi, A. Snyder, M. D. Hellmann, T. Merghoub, J. D. Wolchok, S. A. Shukla, C. J. Wu, K. S. Peggs, T. A. Chan, S. R. Hadrup, S. A. Quezada, C. Swanton, Clonal neoantigens elicit T cell immunoreactivity and sensitivity to immune checkpoint blockade. Science 351, 1463-1469 (2016).

58. E. Eriksson, J. Wenthe, S. Irenaeus, A. Loskog, G. Ullenhag, Gemcitabine reduces MDSCs, tregs and TGF $\beta-1$ while restoring the teff/treg ratio in patients with pancreatic cancer. J. Transl. Med. 14, 282 (2016).

59. Y. Zhang, S. Choksi, K. Chen, Y. Pobezinskaya, I. Linnoila, Z.-G. Liu, ROS play a critical role in the differentiation of alternatively activated macrophages and the occurrence of tumor-associated macrophages. Cell Res. 23, 898-914 (2013).

60. C. Dunnill, T. Patton, J. Brennan, J. Barrett, M. Dryden, J. Cooke, D. Leaper, N. T. Georgopoulos, Reactive oxygen species (ROS) and wound healing: The functional role of ROS and emerging ROS-modulating technologies for augmentation of the healing process. Int. Wound J. 14, 89-96 (2017).
Acknowledgments: We acknowledge L. Huang at UNC for providing the B16F10-luc-GFP and 4T1-luc-GFP cell lines. We acknowledge R. D. Corder and S. A. Khan at NCSU for characterization of hydrogel. We acknowledge the use of the Analytical Instrumentation Facility at NCSU, which was supported by the State of North Carolina and the NSF. Funding: This work was supported by grants from the Alfred P. Sloan Foundation (Sloan Research Fellowship), the North Carolina Translational and Clinical Sciences Institute, the NIH Clinical and Translational Science Awards (NIH grant 1L1TR001111 at UNC-CH to Z.G.), and the pilot grant from the UNC Cancer Center. Author contributions: C.W., J.W., G.D., and Z.G. designed the project. C.W., J.W., and X.Z. performed the experiments and collected the data. All authors analyzed and interpreted the data, contributed to the writing of the manuscript, discussed the results and implications, and edited the manuscript at all stages. Competing interests: Z.G. and C.W. are inventors on patent application (application no.: 62/598,254) held by NCSU that covers design and characterization of the described hydrogel formulation in the paper. All other authors declare that they have no competing interests.

Submitted 3 April 2017

Resubmitted 18 July 2017

Accepted 15 November 2017

Published 21 February 2018

10.1126/scitransImed.aan3682

Citation: C. Wang, J. Wang, X. Zhang, S. Yu, D. Wen, Q. Hu, Y. Ye, H. Bomba, X. Hu, Z. Liu, G. Dotti, $Z$. Gu, In situ formed reactive oxygen species-responsive scaffold with gemcitabine and checkpoint inhibitor for combination therapy. Sci. Transl. Med. 10, eaan3682 (2018). 